\title{
Differential characters and cohomology of the moduli of flat Connections
}

\author{
Marco Castrillón López, Roberto Ferreiro Pérez
}

\begin{abstract}
Let $\pi: P \rightarrow M$ be a principal bundle and $p$ an invariant polynomial of degree $r$ on the Lie algebra of the structure group. The theory of ChernSimons differential characters is exploited to define an homology map $\chi^{k}: H_{2 r-k-1}(M) \times H_{k}(\mathcal{F} / \mathcal{G}) \rightarrow \mathbb{R} / \mathbb{Z}$, for $k<r-1$, where $\mathcal{F} / \mathcal{G}$ is the moduli space of flat connections of $\pi$ under the action of a subgroup $\mathcal{G}$ of the gauge group. The differential characters of first order are related to the Dijkgraaf-Witten action for Chern-Simons Theory. The second order characters are interpreted geometrically as the holonomy of a connection in a line bundle over $\mathcal{F} / \mathcal{G}$ ). The relationship with other constructions in the literature is also analyzed.
\end{abstract}

Mathematics Subject Classification 2010: 53C05, 55R40, 51H25.

Key words and phrases: Characteristic classes, Chern-Simons, differential characters, moduli of flat connections.

Acknowledgements: M.C.L. was partially supported by MINECO (Spain) under grant MTM2015-63612-P.

R.F.P. was partially supported by "Proyecto de investigación SantanderUCM PR26/16-20305”

\section{Introduction}

Given a principal bundle and an invariant polynomials $p$ of the Lie algebra on the structure group, the Chern-Simons differential characters are maps defined on the space of cycles of the base manifold and taking values in $\mathbb{R} / \mathbb{Z}$. Recall that this classical construction is specially relevant when the characteristic classes of the bundle are trivial, since in that case the maps vanish on boundaries and descend to homology with values in $\mathbb{R} / \mathbb{Z}$. They are the so-called secondary Chern-Simons classes. In this paper we study the Chern-Simons differential characters for the principal bundle $(P \times \mathcal{A}) / \mathcal{G} \rightarrow M \times \mathcal{A} / \mathcal{G}$, where $P \rightarrow M$ is any principal bundle, $\mathcal{A}$ is its affine space of connections and $\mathcal{G}$ is any subgroup of the group of gauge transformations of $P$ acting freely on $\mathcal{A}$. The construction is completed, and this is the main result of this article, when we restrict 
the character to the subset $\mathcal{F} \subset \mathcal{A}$ of flat connections. Then we prove that, consistently with the Chern-Simons construction, an homology map

$$
\chi^{k}: H_{2 r-k-1}(M) \times H_{k}(\mathcal{F} / \mathcal{G}) \rightarrow \mathbb{R} / \mathbb{Z},
$$

can be defined any for $k<r-1=\operatorname{deg}(p)$. In the case $k=r-1$ it only gives a map on cycles $\chi^{r-1}: H_{r}(M) \times Z_{r-1}(\mathcal{F} / \mathcal{G}) \rightarrow \mathbb{R} / \mathbb{Z}$ that does not vanish on boundaries.

The final goal of the definition of $\chi^{k}$ is the construction of a tool providing topological information of the moduli space $\mathcal{F} / \mathcal{G}$ of flat connection of $P \rightarrow M$. It is well known the importance of this information in many contexts both in Physics and in Mathematics: bundles modelling quantum phases as that of the Aharonov-Bohm effect, topological Field Theories, moduli spaces of YangMills solution on Riemann surfaces, stability of vector bundles over algebraic varieties, etc. The study of all these situations are present in many works in the literature and cherished by many authors. Our contribution provides a step in that direction.

Along the construction of our main objects, the characteristic classes of $(P \times \mathcal{A}) / \mathcal{G}$ are regarded through the equivariant cohomology of the bundle of connections $C(P) \rightarrow M$. One advantage of working with the equivariant cohomology of $C(P)$ relies on the fact that it is finite-dimensional and we can easily use local coordinates. We also obtain the results by working directly with the equivariant cohomology of $M \times \mathcal{A}$. The bundle of connections is equipped with universal characteristic classes for any invariant polynomial $p$. In particular, this is specially relevant in the case where $2 \operatorname{deg} p>\operatorname{dim} M$, that is, when the corresponding characteristic class vanishes on $M$ because of dimension considerations. In 8, the universal classes are used to obtain maps $H_{2 r-k-1}(M) \times H_{k}(\mathcal{F}) \rightarrow \mathbb{R}$, invariant under the action of the gauge group in $\mathcal{F}$. The invariance induces an homology map for cycles in $\mathcal{F} / \mathcal{G}$ that are projection of cycles in $\mathcal{F}$. Our work generalizes this map to arbitrary elements of $H_{\bullet}(\mathcal{F} / \mathcal{G})$, modulo integers, thus providing a more complete geometric information of the moduli space.

Now we explain how our results are obtained. In $[3$ Chern-Weil theory is applied to the principal $G$-bundle $(P \times \mathcal{A}) / \mathcal{G} \rightarrow M \times \mathcal{A} / \mathcal{G}$. A polynomial $p \in$ $I_{\mathbb{Z}}^{r}(G)$ determines a cohomology class $c_{p} \in H^{2 r}(M \times \mathcal{A} / \mathcal{G})$, and by integrating this class on a closed $d$-dimensional submanifold $c$ of $M$, a cohomology class $\int_{c} c_{p} \in H^{2 r-d}(\mathcal{A} / \mathcal{G})$ is obtained. Moreover, as $p \in I_{\mathbb{Z}}^{r}(G)$, we can also apply the Chern-Simons construction to this bundle. We use the Cheeger-Simons approach of 12 based on differential characters and we denote by $\hat{H}^{k}(M)$ the space of differential characters of order $k$. If $\mathfrak{A}$ is a connection on the principal $\mathcal{G}$-bundle $\mathcal{A} \rightarrow \mathcal{A} / \mathcal{G}$, it determines a connection $\underline{\mathfrak{A}}$ on $(P \times \mathcal{A}) / \mathcal{G} \rightarrow M \times \mathcal{A} / \mathcal{G}$ (see below for details) and hence a differential form $p(\underline{\mathfrak{F}}) \in \Omega^{2 r}(M \times \mathcal{A} / \mathcal{G})$ whose cohomology class is $c_{p}$. As $p \in I_{\mathbb{Z}}^{r}(G)$, there exists a differential character (the Chern-Simons differential character) $\chi_{\mathfrak{A}} \in \hat{H}^{2 r}(M \times \mathcal{A} / \mathcal{G})$ whose curvature is $p(\mathfrak{F})$. As pointed out in 12 a Chern-Simons character is determined by a universal characteristic class $\Upsilon \in H^{2 r}(\mathbf{B} G)$ compatible with $p$. The character 
$\chi_{\underline{\mathfrak{A}}}$ defines maps $\chi_{\underline{\mathfrak{L}}}^{k}: Z_{2 r-k-1}(M) \times Z_{k}(\mathcal{A} / \mathcal{G}) \rightarrow \mathbb{R} / \mathbb{Z}$. We study its restriccion to the moduli space of flat connections and we obtain maps $\chi^{k}: H_{2 r-k-1}(M) \times$ $H_{k}(\mathcal{F} / \mathcal{G}) \rightarrow \mathbb{R} / \mathbb{Z}$ for $k<r-1$, and $\chi^{r-1}: H_{r}(M) \times Z_{r-1}(\mathcal{F} / \mathcal{G}) \rightarrow \mathbb{R} / \mathbb{Z}$ that don't depend on the connection $\mathfrak{A}$ chosen. Hence, for any $c \in Z_{d}(M)$ we define the differential character $\xi_{c, \mathfrak{A}} \in \hat{H}^{2 r-d}(\mathcal{A} / \mathcal{G})$ by $\xi_{c, \mathfrak{A}}(s)=\chi_{\mathfrak{A}}(c \times s)$ and the curvature of $\xi_{c, \underline{A} \mathfrak{I}}$ is $\int_{c} p(\underline{\mathfrak{F}})$. When we restrict to the moduli space of flat connections we obtain cohomology classes $\xi_{c} \in H^{2 r-d-1}(\mathcal{F} / \mathcal{G}, \mathbb{R} / \mathbb{Z})$. We obtain similar results for the moduli space of irreducible flat connections, but using characteristic classes of the group $\tilde{G}=G / Z(G)$, where $Z(G)$ is the center of $G$. We show in Section 8 that these cohomology classes can be related to other constuctions in the literature.

Finally, we study the geometric interpretation of the characters $\xi_{c, \mathfrak{A}}$ of order 1 and 2. The geometrical interpretation of higher order differential characters is not so simple, and we postpone it for future research.

As it is well known, a first order differential character can be interpreted as a function $\mathcal{A} / \mathcal{G} \rightarrow \mathbb{R} / \mathbb{Z}$. In Section 7.1 we identify this function with the Dijkgraaf-Witten action for Chern-Simons theory defined in 13. When restrited to $\mathcal{F} / \mathcal{G}$, we obtain that the Chern-Simons action is locally constant and that it only depends on the homology class of the submanifold $c$.

When $d=2 r-2$ we have $\xi_{c, \underline{A}} \in \hat{H}^{2}(\mathcal{A} / \mathcal{G})$ and, by general results on differential cohomology, it can be represented as the holonomy of a connection on a Hermitian line bundle over $\mathcal{A} / \mathcal{G}$. By fixing a connection $A_{0} \in \mathcal{A}$, we define an explicit principal $U(1)$-bundle $\mathcal{U}_{c} \rightarrow \mathcal{A} / \mathcal{G}$ with connection $\underline{\Theta}_{c}$ and the

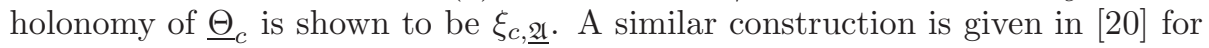
families of connections, with the assumption that the bundles are trivial and working with local trivializations. Our result applies also for non trivial bundles, and we use a global construction.

The character $\xi_{c, \underline{A} \underline{I}}$, the connection $\underline{\Theta}_{c}$ and the action of $\mathcal{G}$ on $\mathcal{A} \times U(1)$ are defined in terms of a connection $\mathfrak{A}$ on the principal $\mathcal{G}$-bundle $\mathcal{A} \rightarrow \mathcal{A} / \mathcal{G}$. However we prove that the action of $\mathcal{G}$ on $\mathcal{A} \times U(1)$ does not depend on the connection $\mathfrak{A}$ chosen.

We study the restriction of this bundle to the moduli space of connections. Then the connection $\underline{\Theta}_{c}$ does not depend on $\mathfrak{A}$. Furthermore,for $r>1$ The holonomy of $\underline{\Theta}_{c}$ only depends on the homology class of $c$. For $r>2$ the connection is flat and the holonomy of $\underline{\Theta}_{c}$ determines the homology class $\xi_{c} \in H^{1}(\mathcal{F} / \mathcal{G}, \mathbb{R} / \mathbb{Z})$.

Along the article, smooth will mean $C^{\infty}, \Omega_{\mathbb{Z}}^{k}$ will denote the space of closed $k$-forms with integral periods, and $\mathcal{I}_{\mathbb{Z}}^{r}(G)$ the algebra of invariant polynomials of degree $r$ on the Lie algebra $\mathfrak{g}$ that are invariant with respect of the adjoint action of $G$ on $\mathfrak{g}$ and determine an integer characteristic class. 


\section{2 preliminaries}

\subsection{Chern-Simons differential characters}

Given an entire form $\alpha \in \Omega_{\mathbb{Z}}^{k}(N)$ and a boundary $c=\partial u$ of a chain $u \in C_{k}(N)$, the integral $\int_{u} \alpha \bmod \mathbb{Z}$ depends only on $c$ and not on $u$, as if $u^{\prime}$ is another chain such that $c=\partial u^{\prime}$, then we have

$$
\int_{u^{\prime}} \alpha=\int_{u} \alpha+\int_{u^{\prime}-u} \alpha=\int_{u} \alpha \bmod \mathbb{Z}
$$

Hence $\alpha$ defines a homomorphism $\chi: B_{k-1}(N) \rightarrow \mathbb{R} / \mathbb{Z}$. Moreover, if $\alpha$ is the characteristic class of a bundle, Chern and Simons shown (see [11]) that it is always possible to extend this map to the space of all cycles on $M$ obtaining a homomorphism $\chi: Z_{k-1}(N) \rightarrow \mathbb{R} / \mathbb{Z}$ such that $\chi(\partial u)=\int_{u} \alpha$ for every $u \in$ $C_{k}(N)$. We now recall this construction (see [1] for details). If $A$ is a connection on a principal $G$-bundle $q: Q \rightarrow N$ with curvature $F$, and $p \in \mathcal{I}_{\mathbb{Z}}^{r}(G)$, we have $p(F) \in \Omega_{\mathbb{Z}}^{k}(N)$. The pull-back of this form to $Q$ is exact $q^{*} p(F)=d T p(A)$, where $T p(A)$ is the Chern-Simons form. Although $T p(A)$ is not projectable onto $N$, its reduction modulo $\mathbb{Z}$ satisfyes

$$
\widetilde{T p(A)}=j^{*} \chi_{A}+d \Lambda
$$

for certain

$$
\chi_{A}: Z_{2 r-1}(N) \rightarrow \mathbb{R} / \mathbb{Z}
$$

and $\Lambda: C_{2 r-2}(Q) \rightarrow \mathbb{R} / \mathbb{Z}$, where $\widetilde{z}$ stands for the image of $z \in \mathbb{R}$ under the map $\mathbb{R} \rightarrow \mathbb{R} / \mathbb{Z}$. The Chern-Simons construction can be interpreted in terms of differential characters (see 12 and 4 for details). A Cheeger-Simons differential character of order $k$ is a homomorphism $\chi: Z_{k-1}(N) \rightarrow \mathbb{R} / \mathbb{Z}$ such that there exist $\alpha \in \Omega^{k}(N)$ with satisfies $\chi(\partial u)=\int_{u} \alpha$ for every $u \in C_{k}(N)$. We say that $\chi$ is a differential character with curvature $\operatorname{curv}(\chi)=\alpha$. We denote the space of differential characters of order $k$ on $N$ by $\hat{H}^{k}(N)$. If $f: N^{\prime} \rightarrow N$ is a smooth map, it induces a map $f^{*}: \hat{H}^{k}(N) \rightarrow \hat{H}^{k}\left(N^{\prime}\right)$ defined by $f^{*} \chi(u)=\chi(f \circ u)$.

In general $\chi_{A}$ is not unique, but it can be seen (e.g. see [12]) that the differential character can be determined uniquely by choosing a universal characteristic class $\Upsilon \in H^{2 r}(\mathbf{B} G, \mathbb{Z})$ on the classifying space $\mathbf{B} G$ compatible with $p$ (i.e. such that $\iota(\Upsilon)=c_{p} \in H^{2 r}(\mathbf{B} G, \mathbb{R})$, where $\iota: H^{2 r}(\mathbf{B} G, \mathbb{Z}) \rightarrow H^{2 r}(\mathbf{B} G, \mathbb{R})$ is the natural map and $c_{p}$ the real universal characteristic class determined by $\left.p \in \mathcal{I}_{\mathbb{Z}}^{r}(G)\right)$. We call $\chi_{A} \in \hat{H}^{2 r}(N)$ the Chern-Simons differential character associated to $p, \Upsilon$ and $A$, and we have $\operatorname{curv}\left(\chi_{A}\right)=p(F)$. If $A^{\prime}$ is another connection on $Q$ we have the following identities

$$
p\left(F_{A^{\prime}}\right)=p\left(F_{A}\right)+d T p\left(A^{\prime}, A\right),
$$

and

$$
\chi_{A^{\prime}}(c)=\chi_{A}(c)+\int_{c} T p\left(A^{\prime}, A\right),
$$


where $\operatorname{Tp}\left(A^{\prime}, A\right)$ is the transgression form

$$
T p\left(A^{\prime}, A\right)=r \int_{0}^{1} p\left(A^{\prime}-A, F_{A_{t}}, \stackrel{(r-1}{\cdots}, F_{A_{t}}\right) d t,
$$

with $A_{t}=(1-t) A^{\prime}+t A$. If $Q^{\prime} \rightarrow N^{\prime}$ is another principal $G$-bundle and $f: Q^{\prime} \rightarrow Q$ is a $G$-bundle morphism we have, $\chi_{f^{*} A}=\underline{f}^{*} \chi_{A}$, where $\underline{f}: N^{\prime} \rightarrow N$ is the induced map on the bases.

Remark 1 The original Chern-Simons and Cheeger-Simons constructions are valid for finite dimensional manifolds, but they can be extended to Banach or Fréchet infinite dimensional manifolds (see [4] for example).

\section{Equivariant Cohomology in the Cartan model}

Let $\mathcal{G}$ be a connected Lie group acting upon a manifold $N$ and let

$$
\Omega_{\mathcal{G}}(N)=\left(\mathbf{S}^{\bullet}\left(\operatorname{Lie} \mathcal{G}^{*}\right) \otimes \Omega^{\bullet}(N)\right)^{\mathcal{G}}=\mathcal{P}^{\bullet}\left(\operatorname{Lie} \mathcal{G}, \Omega^{\bullet}(N)\right)^{\mathcal{G}}
$$

be the space of $\mathcal{G}$-invariat polynomials on Lie $\mathcal{G}$ taking values in $\Omega^{\bullet}(N)$. We consider the graduation in $\Omega_{\mathcal{G}}(N)$ given by $\operatorname{deg}(\alpha)=2 k+r$ for $\alpha \in \mathcal{P}^{k}\left(\operatorname{Lie} \mathcal{G}, \Omega^{r}(N)\right)$, so that the subspace of $\mathcal{G}$-equivariant differential $q$-forms is

$$
\Omega_{\mathcal{G}}^{q}(N)=\bigoplus_{2 k+r=q}\left(\mathcal{P}^{k}\left(\operatorname{Lie} \mathcal{G}, \Omega^{r}(N)\right)\right)^{\mathcal{G}} .
$$

The Cartan equivariant differential $d_{c}: \Omega_{\mathcal{G}}^{q}(N) \rightarrow \Omega_{\mathcal{G}}^{q+1}(N)$ is defined as

$$
\left(d_{c} \alpha\right)(X)=d(\alpha(X))-i_{X_{N}} \alpha(X), \quad \forall X \in \operatorname{Lie} \mathcal{G},
$$

where $X_{N} \in \mathfrak{X}(N)$ is the vector field defined by the infinitesimal action of $X$. It is easy to check that $\left(d_{c}\right)^{2}=0$. The equivariant cohomology in the Cartan model (see for example [7, 21]) of $N$ with respect of the action of $\mathcal{G}$ is defined as the cohomology of this complex.

We now recall the relationship between equivariant cohomology and the cohomology of the quotient space. If the action of $\mathcal{G}$ on $N$ is free and $N / \mathcal{G}$ is a manifold, then $\pi: N \rightarrow N / \mathcal{G}$ is a principal $\mathcal{G}$-bundle. Given a principal connection $A$ in this bundle, we define

$$
\mathrm{C}_{A}(p \otimes \omega)=p\left(F_{A}\right) \wedge \omega_{\text {hor } A},
$$

for any $p \otimes \omega \in\left(\mathbf{S}^{\bullet}\left(\operatorname{Lie} \mathcal{G}^{*}\right) \otimes \Omega^{\bullet}(N)\right)^{\mathcal{G}}$, where $\beta_{\text {hor } A}$ denotes the horizontalization of $\beta \in \Omega^{\bullet}(N)$ with respect to the connection $A$. Extending this map by linearity, we have a sort of Chern-Weil homomorphism

$$
\mathrm{C}_{A}: \Omega_{\mathcal{G}}^{\bullet}(N) \rightarrow\left(\Omega^{\bullet}(N)\right)_{A \text { basic }} \simeq \Omega^{\bullet}(N / \mathcal{G})
$$

satisfying that $\mathrm{C}_{A}\left(d_{c} \alpha\right)=d\left(\mathrm{C}_{A}(\alpha)\right)$. We thus have a map $\mathrm{C}_{A}: H_{\mathcal{G}}^{\bullet}(N) \rightarrow$ $H^{\bullet}(N / \mathcal{G})$, that in fact does not depend on the connection $A$.

We study the map $\mathrm{C}_{A}$ in the two simplest cases: 
Equivariant 1-forms A $\mathcal{G}$-equivariant 1-form is simply a $\mathcal{G}$-invariant 1-form $\alpha$, and $\pi^{*} \mathrm{C}_{A}(\alpha)=\alpha_{\text {hor } A}=\alpha-\alpha(A)$, where $\alpha(A(Y))=\alpha_{y}\left(A(Y)_{N}\right)$ for $Y \in$ $T_{y} N$. The form $\alpha$ is $D$ closed if $d \alpha=0$ and $\iota_{X_{N}} \alpha=0$ for every $X \in$ Lie $\mathcal{G}$. In this case $\alpha$ projects onto a closed 1-form $\underline{\alpha} \in \Omega^{1}(N / \mathcal{G})$. We conclude that if $\alpha$ is $D$-closed, then $\mathrm{C}_{A}(\alpha)=\underline{\alpha}$ does not depend on the connection $A$ chosen.

Equivariant 2-forms A $\mathcal{G}$-equivariant 2-form $\varpi$ is given by $\varpi(X)=\sigma+\mu(X)$ where $\sigma$ is a $\mathcal{G}$-invariant 2-form and $\mu$ : Lie $\mathcal{G} \rightarrow \Omega^{0}(N)$ a linear $\mathcal{G}$-equivariant map. The form $\varpi$ is $D$-closed if $d \sigma=0$ and $i_{X_{N}} \sigma=\mu(X)$ for every $X$. Hence $\mu$ is a co-moment map for $\sigma$. We use below the following result

Lemma 2 If $A$ is a connection on $N \rightarrow N / \mathcal{G}$, we define $\mu(A) \in \Omega^{1}(N)$ by $\mu(A)(Y)=\mu(A(Y))$ for $Y \in T N$, and $\mu(A)$ is $\mathcal{G}$-invariant. If $\varpi=\sigma+\mu$ is $D$-closed then we have $\pi^{*} \mathrm{C}_{A}(\varpi)=\varpi+D(\mu(A))=\sigma+d(\mu(A))$.

Equivariant characteristic classes We also recall here the definition of equivariant characteristic classes of Berline and Vergne (see [5, 6, 9]). Let $\pi: Q \rightarrow N$ a principal $G$-bundle where another Lie group $\mathcal{G}$ acts upon by automorphisms of this bundle. Let $A$ be a connection on $Q$ invariant under the action of $\mathcal{G}$. It can be proved (see [5], 9]) that for every $X \in$ Lie $\mathcal{G}$ the $\mathfrak{g}$-valued function $A\left(X_{P}\right)$ is of adjoint type and defines a section of the adjoint bundle $v_{A}(X) \in \Omega^{0}(N, \operatorname{ad} Q)$. For every $p \in \mathcal{I}^{r}(G)$ the $\mathcal{G}$-equivariant characteristic form associated to $p$ and $A, p_{\mathcal{G}, A} \in \Omega_{\mathcal{G}}^{2 k}(N)$, is defined as

$$
\begin{aligned}
p_{\mathcal{G}, A}(X) & =p\left(F_{A}-v_{A}(X), . \stackrel{(r}{.}, F_{A}-v_{A}(X)\right) \\
& =\sum_{i=0}^{r}(-1)^{r-i}\left(\begin{array}{c}
r \\
i
\end{array}\right) p\left(F_{A}, . \stackrel{(i}{.}, F_{A}, v_{A}(X), \stackrel{(r-i}{*}, v_{A}(X)\right)
\end{aligned}
$$

for every $X \in$ Lie $\mathcal{G}$.

Now we consider the case where $\mathcal{G}$ acts freely on $Q$ and $N$, the quotients $Q / \mathcal{G}$ and $N / \mathcal{G}$ exist and $Q / \mathcal{G} \rightarrow N / \mathcal{G}$ is again a principal $G$-bundle

$$
\begin{array}{ccc}
Q & \stackrel{\bar{\pi}_{\mathcal{G}}}{\rightarrow} & Q / \mathcal{G} \\
\downarrow & & \downarrow \\
N & \stackrel{\pi_{\mathcal{G}}}{\rightarrow} & N / \mathcal{G}
\end{array}
$$

In this case we have the following result.

Proposition 3 If $A_{1}$ is a $\mathcal{G}$-invariant connection on $Q \rightarrow N$ and $A_{2}$ a connection on the (left) $\mathcal{G}$-principal bundle $\pi_{\mathcal{G}}: N \rightarrow N / \mathcal{G}$, then we define the $\mathfrak{g}$-valued 1 -form

$$
A_{1}\left(A_{2}\right)(\xi)=A_{1}\left(\left(A_{2}\left(\pi_{*} \xi\right)\right)_{Q}\right), \quad \xi \in T Q,
$$

Then $A \in \Omega^{1}(Q, \mathfrak{g})$ defined as $A=A_{1}-A_{1}\left(A_{2}\right)$ is projectable to $Q / \mathcal{G}$ and the projection is a connection form $\underline{A}$ on $Q / \mathcal{G} \rightarrow N / \mathcal{G}$. The curvature form of $\underline{A}$ satisfies $\pi_{\mathcal{G}}^{*} F_{\underline{A}}=\left(F_{A_{1}}\right)_{\text {hor } A_{2}}-v_{A_{1}}\left(\pi_{\mathcal{G}}^{*} F_{A_{2}}\right)$. Therefore,

$$
\mathrm{C}_{A_{2}}\left(p_{\mathcal{G}, A_{1}}\right)=p\left(F_{\underline{A}}\right) .
$$


Proof. Given any $X \in$ Lie $\mathcal{G}$, we have

$$
A\left(X_{Q}\right)=A_{1}\left(X_{Q}\right)-A_{1}\left(\left(A_{2}\left(X_{N}\right)\right)_{Q}\right)=A_{1}\left(X_{Q}\right)-A_{1}\left(X_{Q}\right)=0 .
$$

Moreover, $A$ is $\mathcal{G}$-invariant. Indeed, for any $\gamma \in \mathcal{G}$ and any $\xi \in \mathfrak{X}(Q)$, we have

$$
\begin{aligned}
A\left(\gamma_{*} \xi\right) & =A_{1}\left(\gamma_{*} \xi\right)-A_{1}\left(\left(A_{2}\left(\pi_{*} \gamma_{*} \xi\right)\right)_{Q}\right) \\
& =A_{1}\left(\gamma_{*} \xi\right)-A_{1}\left(\left(A_{2}\left(\gamma_{*} \pi_{*} \xi\right)\right)_{Q}\right) \\
& =A_{1}\left(\gamma_{*} \xi\right)-A_{1}\left(\left(\operatorname{Ad}_{\gamma} A_{2}\left(\pi_{*} \xi\right)\right)_{Q}\right) \\
& =A_{1}\left(\gamma_{*} \xi\right)-A_{1}\left(\gamma_{*}\left(A_{2}\left(\pi_{*} \xi\right)\right)_{Q}\right) \\
& =A_{1}(\xi)-A_{1}\left(\left(A_{2}\left(\pi_{*} \xi\right)\right)_{Q}\right)=A(\xi),
\end{aligned}
$$

where we have used the invariance of $A_{1}$ and the fact that $\mathcal{G}$ acts by automorphisms of $Q \rightarrow N$. Then, the form $A$ projects to a one form $\underline{A} \in \Omega^{1}(Q / \mathcal{G}, \mathfrak{g})$.

Given $B \in \mathfrak{g}$, we have

$$
A\left(B_{Q}\right)=A_{1}\left(B_{Q}\right)-A_{1}\left(\left(A_{2}\left(\pi_{*}\left(B_{Q}\right)\right)\right)_{Q}\right)=A_{1}\left(B_{Q}\right)=B,
$$

so that $\underline{A}\left(B_{Q / \mathcal{G}}\right)=B$ as the actions of $\mathcal{G}$ and $G$ commute. Furthermore, the form $A$ is $G$-equivariant. To check that we consider any $g \in G$ and any tangent vector $\xi \in T_{u} Q$. Then

$$
\left(\left(R_{g}\right)^{*} A\right)(\xi)=A_{1}\left(\left(R_{g}\right)_{*}(\xi)\right)-A_{1}\left(\left(A_{2}\left(\pi_{*}\left(R_{g}\right)_{*} \xi\right)\right)_{Q}\right)
$$

where the vector $\left(A_{2}\left(\pi_{*}\left(R_{g}\right)_{*} \xi\right)\right)_{Q}=\left(A_{2}\left(\pi_{*} \xi\right)\right)_{Q}$ has to be evaluated in the point $u g$, as $\left(R_{g}\right)_{*}(\xi) \in T_{u g} Q$. Since the action of $\mathcal{G}$ and $G$ commute, that vector is the right translation of the vector $\left(A_{2}\left(\pi_{*} \xi\right)\right)_{Q}$ evaluated in $u$. That is

$$
\begin{aligned}
\left(\left(R_{g}\right)^{*} A\right)(\xi) & =A_{1}\left(\left(R_{g}\right)_{*}(\xi)\right)-A_{1}\left(\left(R_{g}\right)_{*}\left(A_{2}\left(\pi_{*} \xi\right)\right)_{Q}\right) \\
& =\operatorname{Ad}_{g^{-1}} A_{1}(\xi)-\operatorname{Ad}_{g^{-1}} A_{1}\left(\left(A_{2}\left(\pi_{*}(\xi)\right)\right)_{Q}\right)=\operatorname{Ad}_{g^{-1}} A(\xi) .
\end{aligned}
$$

The commutativity also yields that $\left(R_{g}\right)^{*} \underline{A}=\operatorname{Ad}_{g^{-1}} \underline{A}$, that is, $\underline{A}$ is a connection form.

The horizontal lift $\underline{h}: \mathfrak{X}(N / \mathcal{G}) \rightarrow \mathfrak{X}(Q / \mathcal{G})$ defined by the connection $\underline{A}$ is $\underline{h}(X)=\left(\bar{\pi}_{\mathcal{G}}\right)_{*}\left(h_{1} h_{2} X\right)$, where $h_{1}$ and $h_{2}$ are the horizontal lifts of $A_{1}$ and $A_{2}$ respectively. Indeed, we obviously have that the projection $Q / \mathcal{G} \rightarrow N / \mathcal{G}$ sends $\underline{h} X$ to $X$. On the other hand

$$
\underline{A}(\underline{h} X)=A\left(h_{1} h_{2} X\right)=A_{1}\left(h_{1} h_{2} X\right)-A_{1}\left(\left(A_{2}\left(h_{2} X\right)\right)_{Q}\right)=0,
$$

for any $X \in \mathfrak{X}(N / \mathcal{G})$. Now, the curvature $F_{\underline{A}}$ of $\underline{A}$ understood as a $\mathfrak{g}$-valued 2 -form in $Q / \mathcal{G}$ is

$$
\begin{aligned}
F_{\underline{A}}(\underline{h} X, \underline{h} Y) & =-\underline{A}([\underline{h} X, \underline{h} Y])=-A\left(\left[h_{1} h_{2} X, h_{1} h_{2} Y\right]\right) \\
& =-A_{1}\left(\left[h_{1} h_{2} X, h_{1} h_{2} Y\right]\right)+A_{1}\left(\left(A_{2}\left(\pi_{*}\left[h_{1} h_{2} X, h_{1} h_{2} Y\right]\right)\right)_{Q}\right) \\
& =-A_{1}\left(\left[h_{1} h_{2} X, h_{1} h_{2} Y\right]\right)+A_{1}\left(\left(A_{2}\left(\left[h_{2} X, h_{2} Y\right]\right)\right)_{Q}\right) \\
& =F_{A_{1}}\left(h_{1} h_{2} X, h_{1} h_{2} Y\right)-A_{1}\left(\left(F_{A_{2}}\left(h_{2} X, h_{2} Y\right)\right)_{Q}\right) .
\end{aligned}
$$

If we understood the curvature as a adjoint-valued 2-form, we have the expression in the statement.

Finally, from the definitions of $\mathrm{C}_{A_{2}}$ and $p_{\mathcal{G}, A_{1}}$, the last formula of the statement follows. 


\section{The homology map on the moduli space of flat connections}

\subsection{Chern-Simons characters on $M \times \mathcal{A} / \mathcal{G}$}

Given a principal $G$-bundle $\pi: P \rightarrow M$, let $\mathcal{G} \subset$ Gau $P$ be a subgroup of Gau $P$ such that $\mathcal{G}$ acts freely on the space of connections $\mathcal{A}$ and $\mathcal{A} \rightarrow \mathcal{A} / \mathcal{G}$ is a principal $\mathcal{G}$-bundle. For example we can take $\mathcal{G}$ the subgroup of gauge transformations fixing a point $p_{0} \in P$ (see e.g. [14]).

Remark 4 To be precise, $\mathcal{A}$ and GauP should be defined as the complections of the spaces of connections and Gauge transformations with respect to some Sobolev norms (e.g. see [14]). We do not enter in details because this norms do not play any role in our constructions.

We consider the following diagram of principal bundles

$$
\begin{aligned}
& P \times \mathcal{A} \rightarrow(P \times \mathcal{A}) / \mathcal{G} \\
& \downarrow \quad \downarrow \\
& M \times \mathcal{A} \rightarrow M \times \mathcal{A} / \mathcal{G}
\end{aligned}
$$

where for the vertical projections, the structure group is $G$ acting on the first factor, and the structure group is $\mathcal{G}$ acting on the second factor for the horizontal ones. We regard this diagram as the one given in 4 with $Q=P \times \mathcal{A}$ and $N=M \times \mathcal{A}$ and we are going to apply Proposition 3 .

On one hand, if we consider any connection $\mathfrak{A}$ on the principal $\mathcal{G}$-bundle $\mathcal{A} \rightarrow \mathcal{A} / \mathcal{G}$, we can extend it trivially to $M \times \mathcal{A} \rightarrow M \times \mathcal{A} / \mathcal{G}$ and we denote it by the same letter. On the other hand, $P \times \mathcal{A} \rightarrow M \times \mathcal{A}$ is equipped with a natural connection 1-form $\mathbb{A}$. Indeed, given any $(X, Y) \in T_{(p, A)} P \times \mathcal{A}$, we define $\mathbb{A}((X, Y))=A(X)$. The connection $\mathbb{A}$ is invariant under the acion of the group of automorphisms of $P$,and hence we can define the $\mathcal{G}$-equivariant characteristic forms $p_{\mathcal{G}, \mathbb{A}}$. If $X \in \operatorname{gau} P \simeq \Omega^{0}(M, \operatorname{ad} P)$ we have $v_{\mathbb{A}}(X)=\operatorname{pr}_{1}^{*} X \in$ $\Omega^{0}\left(M \times \mathcal{A}, \operatorname{ad}(P \times \mathcal{A})\right.$, where $\operatorname{pr}_{1}: M \times \mathcal{A} \rightarrow \mathcal{M}$ denotes the projection. Hence we have

$$
p_{\mathcal{G}, \mathbb{A}}(X)=p\left(\mathbb{F}-\operatorname{pr}_{1}^{*} X, \ldots, \mathbb{F}-\operatorname{pr}_{1}^{*} X\right)
$$

According to Proposition 3 , the connections $\mathbb{A}$ and $\mathfrak{A}$ determine a connection $\underline{\mathfrak{A}}$ for the bundle $P \times \mathcal{A} / \mathcal{G} \rightarrow M \times \mathcal{A} / \mathcal{G}$ and we denote by $\mathfrak{F}$ its curvature. Given an invariant polynomial $p \in \mathcal{I}^{r}(G)$, we have the following identity for its associated characteristic form

$$
p(\underline{\mathfrak{F}})=\mathrm{C}_{\mathfrak{A}}\left(p_{\mathcal{G}, \mathbb{A}}\right) \in \Omega_{\mathbb{Z}}^{2 r}(M \times \mathcal{A} / \mathcal{G}) .
$$

We assume that $p \in I_{\mathbb{Z}}^{r}(G)$, that is, $p$ defines an entire characteristic class. We also choose that $\Upsilon \in H^{2 r}(\mathbf{B} G, \mathbb{Z})$ compatible with $p$ and we apply the Chern-Simons construction to the connection $\mathfrak{A}$. We thus get a differential character

$$
\chi_{\underline{\mathfrak{A}}}: Z_{2 r-1}(M \times \mathcal{A} / \mathcal{G}) \rightarrow \mathbb{R} / \mathbb{Z}
$$


determined by $\Upsilon$. Furthermore, we denote by

$$
\chi_{\underline{\mathfrak{A}}}^{k}: Z_{2 r-k-1}(M) \times Z_{k}(\mathcal{A} / \mathcal{G}) \rightarrow \mathbb{R} / \mathbb{Z}
$$

the restriction of the character $\chi_{\mathfrak{\mathfrak { A }}}^{k}(c, s)=\chi_{\underline{\mathfrak{A}}}(c \times s)$ to $(2 r-k-1, k)$ chains.

By definition, we have

$$
\chi_{\underline{\mathfrak{A}}}(\partial K)=\int_{K} p\left(F_{\mathfrak{A}}\right),
$$

for all $K \in C_{2 r}(M \times \mathcal{A} / \mathcal{G})$ and also, for $c \in Z_{2 r-k-1}(M), u \in C_{2 r-k}(M)$, $s \in Z_{k}(\mathcal{A} / \mathcal{G}), t \in C_{k+1}(\mathcal{A} / \mathcal{G})$,

$$
\begin{aligned}
& \chi_{\underline{\mathfrak{A}}}^{k}(\partial u, s)=\int_{u \times s} p(\underline{\mathfrak{F}})=\int_{u \times s} p(\underline{\mathfrak{F}})^{2 r-k, k} \\
& \chi_{\underline{\mathfrak{A}}}^{k}(c, \partial t)=\int_{c \times t} p(\underline{\mathfrak{F}})=\int_{c \times t} p(\underline{\mathfrak{F}})^{2 r-k-1, k+1}
\end{aligned}
$$

\subsection{Restriction to the space of flat connections}

We consider the bigraduation of forms $\Omega^{k}(M \times \mathcal{A}) \simeq \bigoplus_{i=0}^{k} \Omega^{i, j}(M \times \mathcal{A})$ given by the product structure. The bigraduation is extended to equivariant forms by setting $\operatorname{pr}_{1}^{*} X \in \Omega^{0,2}$, i.e. if $\alpha$ : Lie $\mathcal{G} \rightarrow \Omega^{i, j}(M \times \mathcal{A})$ is a polynomial of degree $k$, then $\alpha \in \Omega_{\mathcal{G}}^{i, j+2 k}(M \times \mathcal{A})$. As $\mathfrak{A}$ comes from a connection on $\mathcal{A} \rightarrow \mathcal{A} / \mathcal{G}$, its curvature $\mathfrak{F} \in \Omega^{0,2}(M \times \mathcal{A}, \mathfrak{g})$ and the map $\mathrm{C}_{\mathfrak{\mathscr { A }}}: \Omega_{\mathcal{G}}^{\bullet}(M \times \mathcal{A}) \rightarrow \Omega^{\bullet}(M \times \mathcal{A} / \mathcal{G})$ preserves this bigraduation. Hence we conclude that

$$
p(\underline{\mathfrak{F}})^{i, j}=\mathrm{C}_{\mathfrak{A}}\left(p_{\mathcal{G}, \mathbb{A}}^{i, j}\right) .
$$

The curvature of $\mathbb{A}$ can be decomposed $\mathbb{F}=\mathbb{F}^{2,0}+\mathbb{F}^{1,1}+\mathbb{F}^{0,2}$, where the fact that $\mathbb{F}$ is a form taking values in $\operatorname{ad} P$ instead of a standard form does not make any difference. We use the following result (e.g.see [14, 5.2.3])

Proposition 5 We have $\mathbb{F}^{0,2}=0, \mathbb{F}_{(x, A)}^{1,1}(a, Y)=a(Y)$, and $\mathbb{F}_{(x, A)}^{2,0}\left(Y, Y^{\prime}\right)=$ $F_{A}\left(Y, Y^{\prime}\right)$ for $Y, Y^{\prime} \in T_{x} M$, and $a, a^{\prime} \in T_{A} \mathcal{A} \simeq \Omega^{1}(M, \operatorname{ad} P)$.

The subspace of flat connections $\mathcal{F}=\left\{A \in \mathcal{A}: F_{A}=0\right\} \subset \mathcal{A}$ is invariant under the action of the group $\mathcal{G}$. Let $\iota_{\mathcal{F} / \mathcal{G}}: M \times \mathcal{F} / \mathcal{G} \rightarrow M \times \mathcal{A} / \mathcal{F}$ denote the inclusion.

Proposition 6 We have that

$$
\iota_{\mathcal{F} / \mathcal{G}}^{*}\left(p(\underline{\mathfrak{F}})^{2 r-j, j}\right)=0, \quad \forall j<r .
$$

Proof. By Proposition $\left[\right.$ if $A \in \mathcal{F}$ we have $\mathbb{F}_{(x, A)}^{2,0}=0$ for any $x \in M$. Hence on $\mathcal{F}$ we have $\mathbb{F}=\mathbb{F}^{1,1}$ and by using equation (5) we obtain

$$
\begin{aligned}
\left.p_{\mathcal{G}, \mathbb{A}}\right|_{\mathcal{F}}(X) & =p\left(\mathbb{F}^{1,1}-\operatorname{pr}_{1}^{*} X, \ldots, \mathbb{F}^{1,1}-\operatorname{pr}_{1}^{*} X\right) \\
& =\sum_{i=0}^{r}(-1)^{i}\left(\begin{array}{c}
r \\
i
\end{array}\right) p\left(\operatorname{pr}_{1}^{*} X,{ }^{(i} \cdot, \operatorname{pr}_{1}^{*} X, \mathbb{F}^{1,1}, \stackrel{(r-i}{\cdots}, \mathbb{F}^{1,1}\right) \in \bigoplus_{i=0}^{r} \Omega_{\mathcal{G}}^{r-i, r+i}(M \times \mathcal{A})
\end{aligned}
$$


as $p\left(\operatorname{pr}_{1}^{*} X, \stackrel{(i}{ }^{\prime}, \operatorname{pr}_{1}^{*} X, \mathbb{F}^{1,1}, \stackrel{(r-i}{\cdots}, \mathbb{F}^{1,1}\right) \in \Omega_{\mathcal{G}}^{r-i, r+i}(M \times \mathcal{A})$. In particular, $p_{\mathcal{G}, \mathbb{A}}(X)^{2 r-j, j}=$ 0 , for $j<r$. Together with (11), we conclude (12).

We consider the restriction of the character (8) to the moduli space of flat connections

$$
\left.\chi_{\mathfrak{A}}^{k}\right|_{\mathcal{F} / \mathcal{G}}: Z_{2 r-k-1}(M) \times Z_{k}(\mathcal{F} / \mathcal{G}) \rightarrow \mathbb{R} / \mathbb{Z}
$$

Taking into account (9), (10) and Proposition 6, we have

$$
\left.\chi_{\underline{\mathfrak{A}}}^{k}\right|_{\mathcal{F} / \mathcal{G}}(\partial u, s)=\int_{u \times s} \iota_{\mathcal{F} / \mathcal{G}}^{*} p(\underline{\mathfrak{F}})=\int_{u \times s} \iota_{\mathcal{F} / \mathcal{G}}^{*} p(\underline{\mathfrak{F}})^{2 r-k, k}=0,
$$

for all $u \in C_{2 r-k}(M), s \in Z_{k}(\mathcal{F} / \mathcal{G})$ and $k \leq r-1$. We also have

$$
\left.\chi_{\underline{\mathfrak{A}}}^{k}\right|_{\mathcal{F} / \mathcal{G}}(c, \partial t)=\int_{c \times t} \iota_{\mathcal{F} / \mathcal{G}}^{*} p(\underline{\mathfrak{F}})=\int_{c \times t} \iota_{\mathcal{F} / \mathcal{G}}^{*} p(\underline{\mathfrak{F}})^{2 r-k-1, k+1}=0,
$$

for all $c \in C_{2 r-k-1}(M), t \in Z_{k+1}(\mathcal{F} / \mathcal{G})$ and $k \leq r-2$. Hence for $k \leq r-2$ the $\left.\operatorname{map} \chi_{\underline{\mathfrak{A}}}^{k}\right|_{\mathcal{F} / \mathcal{G}}$ only depends on the homology classes and defines a map

$$
\left.\chi_{\underline{\mathfrak{A}}}^{k}\right|_{\mathcal{F} / \mathcal{G}}: H_{2 r-k-1}(M) \times H_{k}(\mathcal{F} / \mathcal{G}) \rightarrow \mathbb{R} / \mathbb{Z}
$$

And for $k=r-1$ we have a map

$$
\left.\chi_{\underline{\mathfrak{A}}}^{r-1}\right|_{\mathcal{F} / \mathcal{G}}: H_{r}(M) \times Z_{r-1}(\mathcal{F} / \mathcal{G}) \rightarrow \mathbb{R} / \mathbb{Z}
$$

Remark 7 For $k=r-1$ we cannot replace $Z_{r-1}(\mathcal{F} / \mathcal{G})$ by $H_{r-1}(\mathcal{F} / \mathcal{G})$. For example, if $r=2$ we have $\left.\iota_{\mathcal{F} / \mathcal{G}}^{*} \underline{p}_{\mathcal{G}}(X)\right)=p\left(\mathbb{F}^{1,1}-\pi^{*} X, \mathbb{F}^{1,1}-\pi^{*} X\right)=p\left(\mathbb{F}^{1,1}, \mathbb{F}^{1,1}\right)-$ $2 p\left(\pi^{*} X, \mathbb{F}^{1,1}\right)+p\left(\pi^{*} X, \pi^{*} X\right)$, and the term $p\left(\mathbb{F}^{1,1}, \mathbb{F}^{1,1}\right) \in \Omega_{\mathcal{G}}^{2,2}(M \times \mathcal{A})$ is not zero. For example, for $S U(2)$-bundles over a Riemann surface and $p(X)=$ $\frac{1}{8 \pi^{2}} \operatorname{tr}\left(X^{2}\right)$, the form $\sigma=\int_{M} p\left(\mathbb{F}^{1,1}, \mathbb{F}^{1,1}\right)$ gives the symplectic structure on the moduli space of flat connections defined in [2] by $\sigma(a, b)=\frac{1}{4 \pi^{2}} \int_{M} \operatorname{tr}(a \wedge b)$ for $a, b \in \Omega^{1}(M, \operatorname{ad} P)$, and hence $p\left(\mathbb{F}^{1,1}, \mathbb{F}^{1,1}\right)$ is not zero.

Lemma 8 If $\eta \in \Omega^{0,1}(M \times \mathcal{A}, \operatorname{ad}(P \times \mathcal{A}))$ and $F$ is the curvature of the connection $\mathbb{A}+\eta$, then we have $F^{2,0}=\mathbb{F}^{2,0}$. In particular, we have $\underline{\mathfrak{F}}^{2,0}=0$ on $M \times \mathcal{F} / \mathcal{G}$ for any connection $\mathfrak{A}$ on $\mathcal{A} \rightarrow \mathcal{A} / \mathcal{G}$.

Proof. We have $F=\mathbb{F}+d_{\mathbb{A}} \eta+\frac{1}{2}[\eta, \eta]$, and the result follows using that $[\eta, \eta] \in \Omega^{0,2}(M \times \mathcal{A})$, and that $\left(d_{\mathbb{A}} \eta\right)^{2,0}=(d \eta+[\mathbb{A}, \eta])^{2,0}=0$ as $\eta \in \Omega^{0,1}(M \times \mathcal{A})$.

The connections $\mathbb{A}$ and $\bar{\pi}_{\mathcal{G}}^{*} \mathfrak{A}$ are connections on the same bundle $P \times \mathcal{A} \rightarrow$ $M \times \mathcal{A}$. Furthermore, we have $\bar{\pi}_{\mathcal{G}}^{*} \mathfrak{A}=\mathbb{A}+\eta$ with $\eta=-\mathbb{A}(\mathfrak{A})$ for $X \in T(M \times \mathcal{A})$. As $\mathfrak{A}$ comes from a connection on $\mathcal{A} \rightarrow \mathcal{A} / \mathcal{G}$ we have $\eta \in \Omega^{0,1}(M \times \mathcal{A}, \operatorname{ad}(P \times \mathcal{A}))$, and hence $\pi_{\mathcal{G}}^{*} \underline{\mathfrak{F}}^{2,0}=\mathbb{F}^{2,0}=0$ on $M \times \mathcal{F}$. We conclude that $\underline{\mathfrak{F}}^{2,0}=0$.

The Chern-Simons characters are defined using a connection $\mathfrak{A}$ on $\mathcal{A} \rightarrow \mathcal{A} / \mathcal{G}$. Fortunately we have the following result 
Theorem 9 Let $P \rightarrow M$ be a $G$-principal bundle, $p \in \mathcal{I}_{\mathbb{Z}}^{r}(G)$ an invariant polynomial of degree $r, \Upsilon$ a compatible characteristic class and $\mathcal{G} \subset$ Aut $P$ a subgroup acting freely on the space of connections $\mathcal{A}$. Then, following the notation above, the map

$$
\left.\chi_{\underline{\mathfrak{A}}}^{k}\right|_{\mathcal{F} / \mathcal{G}}: Z_{2 r-k-1}(M) \times Z_{k}(\mathcal{F} / \mathcal{G}) \rightarrow \mathbb{R} / \mathbb{Z} .
$$

does not depend on the chosen connection $\mathfrak{A}$ on $\mathcal{A} \rightarrow \mathcal{A} / \mathcal{G}$ for $k<r$.

Proof. Let $\mathfrak{A}, \mathfrak{A}^{\prime}$ be two connections on $\mathcal{A} \rightarrow \mathcal{A} / \mathcal{G}$ and $\underline{\mathfrak{A}}, \underline{\mathfrak{A}}^{\prime}$ the induced connection on the bundle $P \times \mathcal{A} / \mathcal{G} \rightarrow M \times \mathcal{A} / \mathcal{G}$ defined in Proposition 3 ,

By the properties of the Chern-Simons differential characters we have

$$
\chi_{\underline{\mathfrak{A}}^{\prime}}(K)=\chi_{\underline{\mathfrak{A}}}(K)+\int_{K} T p\left(\underline{\mathfrak{A}}^{\prime}, \underline{\mathfrak{A}}\right),
$$

and hence

$$
\chi_{\underline{\mathfrak{A}}^{\prime}}^{k}(c, s)=\chi_{\underline{\mathfrak{A}}}^{k}(c, s)+\int_{c \times s} T p\left(\underline{\mathfrak{A}}^{\prime}, \underline{\mathfrak{A}}\right)=\chi_{\underline{\mathfrak{A}}}^{k}(c, s)+\int_{c \times s} T p\left(\underline{\mathfrak{A}}^{\prime}, \underline{\mathfrak{A}}^{2 r-1-k, k} .\right.
$$

We have $T p\left(\underline{\mathfrak{A}}^{\prime}, \underline{\mathfrak{A}}\right)=r \int_{0}^{1} p\left(\underline{\mathfrak{A}}^{\prime}-\underline{\mathfrak{A}}, F_{t},{ }^{(r-1)}, F_{t}\right) d t$ with $\eta=\underline{\mathfrak{A}}^{\prime}-\underline{\mathfrak{A}}$ and $F_{t}$ the curvature of $(1-t) \underline{\mathfrak{A}}^{\prime}+t \underline{\mathfrak{A}}$. As $\mathfrak{A}$ and $\mathfrak{A}^{\prime}$ come from connections on $\mathcal{A} \rightarrow \mathcal{A} / \mathcal{G}$ we have $\underline{\mathfrak{A}}^{\prime}-\underline{\mathfrak{A}} \in \Omega^{0,1}(M \times \mathcal{A} / \mathcal{G}, \operatorname{ad}(P \times \mathcal{A} / \mathcal{G}))$. By Lemma 8 we have $F_{t}^{2,0}=0$ on $M \times \overline{\mathcal{F}} / \mathcal{G}$. Hence $T p\left(\underline{\mathfrak{A}}, \underline{\mathfrak{A}}^{\prime}\right)^{2 r-1-k, k}=0$ for $k<r$, and the proof is complete.

Since the map $\left.\chi_{\mathfrak{a}}^{k}\right|_{\mathcal{F} / \mathcal{G}}$ does not depend on the connection $\mathfrak{A}$, we simplify the notation and directly write

$$
\chi^{k}: H_{2 r-k-1}(M) \times H_{k}(\mathcal{F} / \mathcal{G}) \rightarrow \mathbb{R} / \mathbb{Z}, \quad k \leq r-2,
$$

and

$$
\chi^{r-1}: H_{r}(M) \times Z_{r-1}(\mathcal{F} / \mathcal{G}) \rightarrow \mathbb{R} / \mathbb{Z},
$$

as the result of the constructions developed in this section.

Finally, the following result shows that $\chi^{k}(c, s)$ coincides with the homology map defined in 8 when $s \in Z \bullet(\mathcal{F} / \mathcal{G})$ is a projection of a cycle $\bar{s}$ in $\mathcal{F}$. We denote by $\pi_{\mathcal{G}}: M \times \mathcal{A} \rightarrow M \times \mathcal{A} / \mathcal{G}$ the projection.

Proposition 10 For $c \in Z_{2 r-1-k}(M)$ and $\bar{s} \in Z_{k}(\mathcal{F})$, let $\bar{t} \in C_{k+1}(\mathcal{A})$ be a chain that satisfies $\partial \bar{t}=\bar{s}$ (it exists because $\mathcal{A}$ is contractible) and set $s=$ $\pi_{\mathcal{G}} \circ \bar{s} \in Z_{k}(\mathcal{F} / \mathcal{G})$. Then we have

$$
\chi^{k}(c, s)=\int_{c \times \bar{t}} p(\mathbb{F}), \quad k<r .
$$

Proof. We define $t=\pi_{\mathcal{G}} \circ \bar{t} \in C_{k+1}(\mathcal{A} / \mathcal{G})$ so that $\partial t=s$. As the connections $\mathbb{A}$ and $\pi_{\mathcal{G}}^{*} \mathfrak{A}$ are connections on the same bundle $P \times \mathcal{A} \rightarrow M \times \mathcal{A}$, we have 
$p(\mathbb{F})-\pi_{\mathcal{G}}^{*} p(\underline{\mathfrak{F}})=d\left(T p\left(\mathbb{A}, \pi_{\mathcal{G}}^{*} \mathfrak{A}\right)\right)$, and using equation (10) we obtain

$$
\begin{aligned}
\chi_{\underline{\mathfrak{A}}}^{k}(c, s) & =\chi_{\underline{\mathfrak{A}}}^{k}(c, \partial t)=\int_{c \times t} p\left(F_{\underline{\mathfrak{A}}}\right)=\int_{c \times \bar{t}} \pi_{\mathcal{G}}^{*} p\left(F_{\underline{\mathfrak{A}}}\right) \\
& =\int_{c \times \bar{t}} p(\mathbb{F})+\int_{c \times \bar{s}} T p\left(\mathbb{A}, \pi_{\mathcal{G}}^{*} \mathfrak{A}\right)=\int_{c \times \bar{t}} p(\mathbb{F})+\int_{c \times \bar{s}} T p\left(\mathbb{A}, \pi_{\mathcal{G}}^{*} \mathfrak{A}\right)^{2 r-1-k, k} .
\end{aligned}
$$

We have $T p\left(\mathbb{A}, \pi_{\mathcal{G}}^{*} \mathfrak{A}\right)=r \int_{0}^{1} p\left(\eta, F_{t},{ }^{(r-1)}, F_{t}\right) d t$ with $F_{t}$ the curvature of $\mathbb{A}+t \eta$. By Proposition 3 we have $\pi_{\mathcal{G}}^{*} \mathfrak{A}=\mathbb{A}+\eta$ with $\eta=-\mathbb{A}(\mathfrak{A})$ for $X \in T(M \times \mathcal{A})$. As $\mathfrak{A}$ comes from a connection on $\mathcal{A} \rightarrow \mathcal{A} / \mathcal{G}$ we have $\eta \in \Omega^{0,1}(M \times \mathcal{A}, \operatorname{ad}(P \times \mathcal{A}))$. By Lemma 8 it follows that $F_{t}^{2,0}=0$ on $M \times \mathcal{F}$, and hence $T p\left(\mathbb{A}, \pi_{\mathcal{G}}^{*} \mathfrak{A}\right)^{2 r-1-k, k}=0$ for $k<r$.

\section{Construction using the bundle of connections}

In the preceding section our basic result Proposition 6 is proved using the expression of the curvature $\mathbb{F}$ in the infinite dimensional manifold $M \times \mathcal{A}$. In this Section we show that in accordance with the results of [16] Proposition 6 ] can be also obtained by studying the equivariant characteristic classes of the finite dimensional bundle of connections $C(P)$. In our case both approaches are equivalent, but we recall that for example, in the study of locality in anomaly cancellation it is fundamental the fact the equivariant forms can be obtained from the bundle of connections (see [18] for details).

\subsection{Bundle of connections}

Given a fiber bundle $\pi: E \rightarrow M$, an Ehresmann connection is a section of the jet bundle $J^{1} E \rightarrow E$ of local sections of $E \rightarrow M$. Geometrically, this section $\varsigma: E \rightarrow J^{1} E$ assigns to every point $y \in E$, a complementary subspace $H_{y} E=s_{*}\left(T_{x} M\right) \subset T_{y} E$, of the vertical subspace $V_{y} E \subset T_{y} E$, where $\varsigma(y)=$ $j_{x}^{1} s, s(x)=y$ and $V_{y} E=\operatorname{ker} \pi_{*}$. If the bundle $E \rightarrow M$ is a $G$-principal bundle $P \rightarrow M$, connections are assumed to be $G$-invariant in the sense that $\left(R_{g}\right)_{*} H_{y} P=H_{R_{g}(y)} P$, for every $g \in G$, where $R_{g}: P \rightarrow P$ is the right action of $G$ on $P$. Therefore, principal connections are sections of the quotient bundle $\left(J^{1} P\right) / G \rightarrow P / G=M$, where the action $R_{g}$ lifts to the jet space in the natural way, i.e., $R_{g}\left(j_{x}^{1} s\right)=j_{x}^{1}\left(R_{g} \circ s\right)$. This bundle is called the bundle of connections and is denoted by $q: C(P) \rightarrow M$.

Since, on one hand, $J^{1} P \rightarrow P$ is an affine bundle modeled by the vector bundle $T^{*} M \otimes V P \rightarrow P$ and, on the other hand, we have a natural identification

$$
P \times \mathfrak{g}=V P,\left.\quad(y, \xi) \mapsto \frac{d}{d \epsilon}\right|_{\epsilon=0} R_{\exp \epsilon \xi} y,
$$

the bundle of connections is also an affine bundle, modeled by the vector bundle $T^{*} M \otimes \operatorname{ad} P \rightarrow M$, where $\operatorname{ad} P=(P \times \mathfrak{g}) / G$ is the adjoint bundle, that is, the 
associated bundle to $P$ by the adjoint action of $G$ on its Lie algebra $\mathfrak{g}$. Then we have that $\operatorname{dim} C(P)=n+n m, n=\operatorname{dim} M, m=\operatorname{dim} G$.

The principal $G$-bundle $J^{1} P \rightarrow C(P)$ is equipped with a canonical (tautological) principal connection form $\mathbf{A}$ (for example, see [10]). This form is the contact form of the jet bundle defined as $\mathbf{A}(v)=\xi \in \mathfrak{g}, v \in T_{j_{x}^{1} s}\left(J^{1} P\right)$, where $\xi$ is the element of the Lie algebra associated to $\left(\pi_{10}\right)_{*} v-\left(s \circ \pi_{1}\right)_{*} v \in V_{s(x)} P$ by the isomorphism (16), and the source and target projections of the jet bundle are $\pi_{1}: J^{1} P \rightarrow M, \pi_{10}: J^{1} P \rightarrow P$ respectively. The connection form $\mathbf{F}$ of $\mathbf{A}$ can be seen as a 2-form in $C(P)$ taking values in the adjoint bundle of $J^{1} P \rightarrow C(P)$. It is easy to check that the bundles $q^{*} P \rightarrow C(P)$ and $J^{1} P \rightarrow C(P)$ are naturally diffeomorphic, so that $\mathbf{F} \in \Omega\left(C(P), q^{*}\right.$ ad $\left.P\right)$. This canonical curvature form satisfies that, given a section $\sigma: M \rightarrow C(P)$ of the bundle of connections, that is, a connection $A_{\sigma}$ on $P \rightarrow M$, the pull-back $\sigma^{*}(\mathbf{F}) \in \Omega^{2}(M, \operatorname{ad} P)$ is the curvature 2-form $F_{A_{\sigma}}$ of $A_{\sigma}$.

Coordinates in $C(P)$ are defined as follows. Let $\left(x^{1}, \ldots, x^{n}\right)$ be a coordinate system on a domain $U \subset M$ where the principal bundle is trivializable, $\pi^{-1}(U)=U \times G$. The trivialization induces a (flat) connection on $\pi^{-1}(U) \rightarrow U$ and therefore a section $\sigma_{U}:\left.U \rightarrow C(P)\right|_{U}$ of the bundle of connection. With this section, we identify the affine bundle $\left.C(P)\right|_{U} \rightarrow U$ with $\left.\left(T^{*} M \otimes \operatorname{ad} P\right)\right|_{U} \rightarrow U$. If $\left(B_{1}, \ldots, B_{m}\right)$ is a basis of $\mathfrak{g}$, the set $\left(\tilde{B}_{1}, \ldots, \tilde{B}_{m}\right)$ is a basis of section of $\left.\operatorname{ad} P\right|_{U} \rightarrow U$, where $\tilde{B}_{i}(x)=\left((x, g), \operatorname{Ad}_{g} B_{i}\right)_{G} \in \operatorname{ad} P_{x}, x \in U$. Coordinates $\left(A_{j}^{\alpha}\right), j=1, \ldots, n, \alpha=1, \ldots, m$, are defined as $A=A_{j}^{\alpha}(A) d x^{j} \otimes \tilde{B}_{\alpha}$ for any $\left.\left.A \in T^{*} M \otimes \operatorname{ad} P\right|_{U} \simeq C(P)\right|_{U}$. It is not very complicated to check (for instance, see [10]) that in this coordinate system the canonical curvature from takes the expression

$$
\mathbf{F}=\left(d A_{j}^{\alpha} \wedge d x^{j}+c_{\beta \gamma}^{\alpha}\left(A_{i}^{\beta} A_{j}^{\gamma}-A_{j}^{\beta} A_{i}^{\gamma}\right) d x^{i} \wedge d x^{j}\right) \otimes \tilde{B}_{\alpha} .
$$

Given any fiber bundle $\pi: E \rightarrow M$, a differential form $\eta \in \Omega^{\bullet}\left(J^{1} E\right)$ is said to be horizontal or 0 -contact if $i_{Y} \eta=0$ for every vector $Y \in T\left(J^{1} E\right)$ vertical with respect to the projection $\pi_{1}: J^{1} E \rightarrow M$. On the other hand, $\eta$ is said to be contact if $\left(j^{1} s\right)^{*} \eta=0$, for every (local) section $s$ of $\pi$. Given any $r$-form $\omega$ on $E$, its pull-back $\pi_{10}^{*} \omega \in \Omega^{\bullet}\left(J^{1} E\right)$ can be decomposed as the sum of a horizontal and a contact form in a unique way. This splitting can be further refined. A contact form is said to be $k$-contact if for every vertical vector $Y$, the form $i_{Y} \omega$ is $(k-1)$-contact. Then, as proved in [23, every $r$-form $\omega \in \Omega^{\bullet}(E)$ admits a unique decomposition

$$
\pi_{10}^{*} \omega=\sum_{s=0}^{r} \omega^{s, r-s}
$$

where $\omega^{s, r-s} \in \Omega^{\bullet}\left(J^{1} E\right)$ is $s$-contact. In the case where $E=C(P)$, it is easy 
to check that the decomposition of the canonical curvature $\mathbf{F}$ reads

$$
\begin{aligned}
& \mathbf{F}^{2,0}=\sum_{i<j}\left(A_{j, i}^{\alpha}-A_{i, j}^{\alpha}+c_{\beta \gamma}^{\alpha}\left(A_{i}^{\beta} A_{j}^{\gamma}-A_{j}^{\beta} A_{i}^{\gamma}\right)\right) d x^{i} \wedge d x^{j} \otimes \tilde{B}_{\alpha} \\
& \mathbf{F}^{1,1}=\left(d A_{i}^{\alpha}-A_{i, j}^{\alpha} d x^{j}\right) \wedge d x^{i} \otimes \tilde{B}_{\alpha} \\
& \mathbf{F}^{0,2}=0
\end{aligned}
$$

where the fact that $\mathbf{F}$ is a form taking values in $q^{*}$ ad $P$ instead of a standard form does not make any difference.

We consider the evaluation map

$$
\begin{aligned}
\mathrm{ev}: M \times \mathcal{A} & \rightarrow C(P) \\
(x, A) & \mapsto \sigma_{A}(x) .
\end{aligned}
$$

Proposition 11 Let $\mathbf{A}$ be the canonical connection on the bundle $J^{1} P \rightarrow$ $C(P)$. For any subgroup $\mathcal{G} \subset$ Aut $P$, we consider its natural action on $J^{1} P \rightarrow$ $C(P)$. Then, we have the following identity of equivariant characteristic forms

$$
\mathrm{ev}^{*} p_{\mathcal{G}, \mathbf{A}}=p_{\mathcal{G}, \mathbb{A}}
$$

Proof. The map ev is AutP-equivariant so that it defines a map

$$
\mathrm{ev}^{*}: \Omega_{\mathcal{G}}^{\bullet}(C(P)) \rightarrow \Omega_{\mathcal{G}}^{\bullet}(M \times \mathcal{A}) .
$$

If we consider another evaluation map $\overline{\mathrm{ev}}: P \times \mathcal{A} \rightarrow J^{1} P, \overline{\mathrm{ev}}(p, A)=\operatorname{Hor}_{p}^{A}$, where $\operatorname{Hor}_{p}^{A}: T_{x} M \rightarrow T_{p} P, \pi(p)=x$, is the horizontal lift to $p$ defined by $A$, we have the diagram



It is easy to check that the pullback of the connection form $\overline{\mathrm{ev}}^{*} \mathbf{A}$ is exactly the connection form $\mathbb{A}$ in $P \times \mathcal{A} \rightarrow M \times \mathcal{A}$ introduced above. This completes the proof.

The evaluation map can be naturally extended to the jet space $J^{1} C(P)$

$$
\begin{aligned}
\mathrm{ev}_{1} & : M \times \mathcal{A} \rightarrow J^{1} C(P) \\
(x, A) & \mapsto j_{x}^{1} A
\end{aligned}
$$

and (e.g. see [17]) the map $\mathrm{ev}_{1}$ is compatible with the bigraduation of forms on $M \times \mathcal{A}$ given by the product structure and the bigraduation $\Omega^{i, j}(C(P))$ on $J^{1} C(P)$ of contact forms, that is,

$$
\mathrm{ev}_{1}^{*}\left(\Omega^{i, j}(C(P)) \subset \Omega^{i, j}(M \times \mathcal{A}) .\right.
$$

The bigraduation is extended to equivariant forms by setting $\pi^{*} X \in \Omega^{0,2}$, i.e. if $\alpha: \operatorname{Lie} \mathcal{G} \rightarrow \Omega^{i, j}(C(P))$ is a polynomial of degree $k$, then $\alpha \in \Omega_{\mathcal{G}}^{i, j+2 k}(C(P))$, 
and the same definition for forms on $M \times \mathcal{A}$. As $\mathfrak{A}$ comes from a connection on $\mathcal{A} \rightarrow \mathcal{A} / \mathcal{G}$ the map $\mathrm{C}_{\underline{\mathfrak{A}}}: \Omega_{\mathcal{G}}^{\bullet}(M \times \mathcal{A}) \rightarrow \Omega^{\bullet}(M \times \mathcal{A} / \mathcal{G})$ also preserves this bigraduation. Hence we conclude that $p(\underline{\mathfrak{F}})^{i, j}=\mathrm{C}_{\mathfrak{A}}\left(\mathrm{ev}_{1}^{*} p_{\mathcal{G}, \mathbf{A}}^{i, j}\right)$.

We consider the subset $\mathcal{E}_{\mathcal{F}}=\left\{j_{x}^{1} A \in J^{1} C(P): \mathbf{F}_{j_{x}^{1} A}^{2,0}=0\right\} \subset J^{1} C(P)$ of 1-jets of flat connections. Obviously, a connection $A \in \Gamma(M, C(P))$ is flat if and only if $j_{x}^{1} A \in \mathcal{E}_{\mathcal{F}}$ for every $x \in M$. On $\mathcal{E}_{\mathcal{F}}$ we have

$$
\begin{aligned}
& p_{\mathcal{G}, \mathbf{A}} \mid \mathcal{E}_{\mathcal{F}}(X)=p\left(\mathbf{F}^{1,1}-\pi^{*} X, \ldots, \mathbf{F}^{1,1}-\pi^{*} X\right) \\
& =\sum_{i=0}^{r}(-1)^{i}\left(\begin{array}{l}
r \\
i
\end{array}\right) p\left(\pi^{*} X, \stackrel{(i}{i}^{\prime}, \pi^{*} X, \mathbf{F}^{1,1}, \stackrel{(r-i}{\cdots}, \mathbf{F}^{1,1}\right) \in \bigoplus_{i=0}^{r} \Omega^{r-i, r+i}(C(P))
\end{aligned}
$$

as $p\left(\pi^{*} X, \stackrel{(i}{i}^{.}, \pi^{*} X, \mathbf{F}^{1,1}, \stackrel{(r-i}{\cdot}, \mathbf{F}^{1,1}\right) \in \Omega^{r-i, r+i}(C(P))$. In particular, $p_{\mathcal{G}, \mathbf{A}}(X)^{2 r-j, j}=$ 0 , for $j<r$, and Proposition 6 follows.

\section{Cohomology classes on the moduli space of irreducible flat connections}

If we denote by $\widetilde{\mathcal{A}}$ the space of irreducible connections, then $\widetilde{\mathcal{A}} / \mathrm{Gau} P$ is a well defined manifold and we can try to apply the preceding construction to this case. The unique obstacle to do it is that the action of $\operatorname{Gau} P$ on $\widetilde{\mathcal{A}}$ is not free. If we denote by $Z(G)$ the center of $G$, then the isotropy of any $A \in \widetilde{\mathcal{A}}$ is $Z(G)$ (considered as constant gauge transformations). Hence, if we define the group $\widetilde{\mathcal{G}}=\operatorname{Gau} P / Z(G)$ then $\widetilde{\mathcal{G}}$ acts freely on $\widetilde{\mathcal{A}}$ and $\widetilde{\mathcal{A}} \rightarrow \widetilde{\mathcal{A}} / \widetilde{\mathcal{G}}$ is a principal $\tilde{\mathcal{G}}$-bundle (see [14]). Note that $\widetilde{\mathcal{A}} / \widetilde{\mathcal{G}}=\widetilde{\mathcal{A}} / \mathrm{Gau} P$ an hence this quotient is the moduli space of irreducible connections. The problem now is that $\widetilde{\mathcal{G}}$ does not act on $P \times \widetilde{\mathcal{A}}$ because $Z(G)$ does not act trivially on $P$. To solve this problem we define $\widetilde{\mathbb{P}}=(P \times \widetilde{\mathcal{A}}) / Z(G)=(P / Z(G)) \times \widetilde{\mathcal{A}}$ which is a principal $\widetilde{G}$-bundle, where $\tilde{G}=G / Z(G)$.

Let $\mathfrak{z}$ be the lie algebra of $Z(G)$ and $\widetilde{\mathfrak{g}}=\mathfrak{g} / \mathfrak{z}$ the Lie algebra of $\widetilde{G}$. We denote by $t: \mathfrak{g} \rightarrow \widetilde{\mathfrak{g}}$ the projection. The connection $\mathbb{A} \in \Omega^{1}(P \times \mathcal{A}, \mathfrak{g})$ induces a connection $\widetilde{\mathbb{A}}$ on $\widetilde{\mathbb{P}}$ which is invariant under the action of $\widetilde{\mathcal{G}}$ in the following way.

Lemma 12 The form $t \circ \mathbb{A} \in \Omega^{1}(P \times \mathcal{A}, \widetilde{\mathfrak{g}})$ projects to a connection form $\widetilde{\mathbb{A}} \in$ $\Omega^{1}(\widetilde{\mathbb{P}}, \widetilde{\mathfrak{g}})$ which is invariant under the action of $\widetilde{\mathcal{G}}$.

Proof. On one hand, given $B \in \mathfrak{z}$, we have $t \circ \mathbb{A}\left(B_{\mathbb{P}}\right)=t(B)=0$. On the other, for $g \in Z(G), R_{g}^{*}(t \circ \mathbb{A})=t \circ R_{g}^{*} \mathbb{A}=t \circ \operatorname{Ad}_{g^{-1}} \mathbb{A}=t \circ \mathbb{A}$. Hence $t \circ \mathbb{A}=\pi_{Z}^{*} \tilde{\mathbb{A}}$, where $\pi_{Z}: \mathbb{P} \rightarrow \tilde{\mathbb{P}}$. As the fundamental vector fields $B_{\mathbb{P}}, B \in \mathfrak{g}$, project to $(t(B))_{\tilde{\mathbb{P}}}$, we have that $\tilde{\mathbb{A}}\left(D_{\tilde{\mathbb{P}}}\right)=D$, for any $D \in \widetilde{\mathfrak{g}}$. Moreover, given $(g)_{Z} \in \tilde{G}$, since $t \circ \operatorname{Ad}_{g}=\operatorname{Ad}_{(g)_{Z}} \circ t$, we have that $R_{(g)_{Z}}^{*} \tilde{\mathbb{A}}=\operatorname{Ad}_{(g)_{Z}^{-1}} \tilde{\mathbb{A}}$, and $\tilde{\mathbb{A}}$ is a connection form.

Finally, since the actions of $G$ and $\mathcal{G}$ commute on $P \times \mathcal{A}$, the invariance of $\mathbb{A}$ with respect to $\mathcal{G}$ induces the invariance of $\tilde{\mathbb{A}}$ with respect to $\tilde{\mathcal{G}}$. 
Now we can apply all the preceding results, but we should take $p \in \mathcal{I}_{\mathbb{Z}}^{r}(\tilde{G})$ and $\mu \in H^{2 r}(B \widetilde{G}, \mathbb{Z})$ in place of the corresponding objects for $G$.

As $\widetilde{\mathbb{A}}$ is $\widetilde{\mathcal{G}}$-invariant, if $\mathfrak{A}$ is a connection on $\widetilde{\mathcal{A}} \rightarrow \widetilde{\mathcal{A}} / \widetilde{\mathcal{G}}$, by Proposition 3 they determine a connection on the quotient bundle $\widetilde{\mathbb{P}} / \widetilde{\mathcal{G}} \rightarrow M \times \widetilde{\mathcal{A}} / \widetilde{\mathcal{G}}$, and we have the Chern-Simons characters $\widetilde{\chi}_{\mathfrak{A}}: Z_{2 r-1}(M \times \widetilde{\mathcal{A}} / \widetilde{\mathcal{G}}) \rightarrow \mathbb{R} / \mathbb{Z}$. Doing the same as in Section 4 we obtain maps $\widetilde{\chi}_{\mathfrak{A}}^{k}: Z_{2 r-k-1}(M) \times Z_{k}(\widetilde{\mathcal{A}} / \widetilde{\mathcal{G}}) \rightarrow \mathbb{R} / \mathbb{Z}$.

If we restrict to $\widetilde{\mathcal{F}}=\left\{A \in \mathcal{A}: F_{A}=0, A\right.$ is irreducible $\} \subset \widetilde{\mathcal{A}}$ the space of irreducible flat connections, using the the same arguments as in Theorem 9 we get cohomology maps $\widetilde{\chi}^{k}: H_{2 r-k-1}(M) \times H_{k}(\widetilde{\mathcal{F}} / \widetilde{\mathcal{G}}) \rightarrow \mathbb{R} / \mathbb{Z}$ that does not depend on the connection $\mathfrak{A}$

We recall that if $G=U(n)$ or $S U(n)$, then $\widetilde{G}=P U(n)=S U(n) / \mathbb{Z}_{n}$ is the projective unitary group. We have $\mathfrak{s u}(n)=\mathfrak{p u}(n)$ and $\mathcal{I}^{r}(P U(n))=\mathcal{I}^{r}(S U(n))$ as they are connected groups. However, we have $\mathcal{I}_{\mathbb{Z}}^{r}(P S U(n)) \varsubsetneqq \mathcal{I}_{\mathbb{Z}}^{r}(S U(n))$. The projection $i: S U(n) \rightarrow P U(n)$ induces a map $B i^{*}: H^{\bullet}(\mathbf{B} P U(n), \mathbb{Z}) \rightarrow$ $H^{\bullet}(\mathbf{B} S U(n), \mathbb{Z})$. It is shown in 1 that $H^{4}(\mathbf{B} P U(n), \mathbb{Z}) \simeq \mathbb{Z}$ has a generator $e_{2}$ such that $B i^{*}\left(e_{2}\right)=2 n c_{2}$ for $n$ even and $B \rho^{*} e_{2}=n c_{2}$ for $n$ odd, where $c_{2}$ denotes the second Chern class. Hence if $C_{2} \in \mathcal{I}_{\mathbb{Z}}^{2}(S U(n))$ is the second Chern polynomial we have $C_{2} \notin \mathcal{I}_{\mathbb{Z}}^{2}(P U(n))$ but $2 n C_{2} \in \mathcal{I}_{\mathbb{Z}}^{2}(P U(n))$. We conclude that for a $S U(n)$-principal bundle $\left(C_{2}, c_{2}\right)$ does not determine cohomology classes on $\widetilde{\mathcal{F}} / \widetilde{\mathcal{G}}$, but $\left(2 n C_{2}, e_{2}\right)$ does.

\section{Low dimensional cases}

If $c \in Z_{2 r-k-1}$, we define $\xi_{c, \underline{A}} \in \hat{H}^{k+1}(\mathcal{A} / \mathcal{G})$ by $\xi_{c, \underline{A}}(s)=\chi_{\mathfrak{L}}^{k}(c \times s)$. In this Section we study the geometrical interpretation of the differential characters $\xi_{c, \mathfrak{A} \text { f }}$ for $k=0$ and $k=1$.

\subsection{First order characters and Dijkgraaf-Witten action for Chern-Simons theory}

We recall the definition of the Dijkgraaf-Witten action for Chern-Simons theory. Usually classical Chern-Simons theory is defined for trivial bundles over 3 -manifolds using global sections, but this procedure can not be generalized to non-trivial bundles. In [13] Dijkgraaf and Witten shown how the Chern-Simons action can be defined in this case using differential characters.

Let $A$ be a connection on $P \rightarrow M, p \in \mathcal{I}_{\mathbb{Z}}^{r}(G), \Upsilon$ a compatible characteristic class and let $\chi_{A} \in \hat{H}^{2 r}(M)$ be the Chern-Simons character of $A$ associated to $p$ and $\Upsilon$. If $c: C \rightarrow M$ is a smooth map with $C$ closed and $\operatorname{dim} C=2 r-1$ we define the Chern-Simons action $\lambda_{c}: \mathcal{A} \rightarrow \mathbb{R} / \mathbb{Z}$ by $\lambda_{c}(A)=\chi_{A}(c)$. By definition, if $c=\partial u$ we have $\lambda_{\partial u}(A)=\chi_{A}(\partial u)=\int_{u} p(F, \ldots, F)$. If $A_{0}$ is another connection on $P$ we have $\lambda_{c}(A)-\lambda_{c}\left(A_{0}\right)=\chi_{A}(c)-\chi_{A_{0}}(c)=\int_{c} T p\left(A, A_{0}\right)$ and hence $\lambda_{c}(A)=\lambda_{c}\left(A_{0}\right)+\int_{c} T p\left(A, A_{0}\right)$. Note that $\lambda_{c}$ gives a definition of the ChernSimons action which is independent of the background connection $A_{0}$ chosen, 
i.e., the characteristic class $\Upsilon$ fixes the value of $\lambda_{c}\left(A_{0}\right)$. As remarked in [13] this constant is relevant in the quantization of the theory. It can be seen that $\lambda_{c}$ is $\mathcal{G}$-invariant.

Now we show how first order characters are related to the Chern-Simons Lagrangian $\lambda_{c}$.

A differential character of order 1 is a homomorphism $\chi: Z_{0}(M) \rightarrow \mathbb{R} / \mathbb{Z}$ such that there exist $\lambda \in \Omega^{1}(M)$ with $\chi(\{x\}-\{y\})=\int_{\gamma} \lambda$ for any $\gamma \subset M$, $\partial \gamma=x-y$. We define $\varphi_{\chi}: M \rightarrow \mathbb{R} / \mathbb{Z}$ by $\varphi_{\chi}(x)=\chi(\{x\})$. It can be seen that $\varphi$ is differentiable (see [4]). Conversely, given any map $\varphi: M \rightarrow \mathbb{R} / \mathbb{Z}$, we can define $\chi_{\varphi}: Z_{0}(M) \rightarrow \mathbb{R} / \mathbb{Z}$ by setting $\chi_{\varphi}(\{x\})=\varphi(x)$, and $\lambda=\varphi^{*}(d t)$ where $t$ is a coordinate on $\mathbb{R}$. Hence, a differential character of order 1 is simply a map $\varphi: M \rightarrow \mathbb{R} / \mathbb{Z}$.

Lemma 13 The character $\xi_{c, \underline{A}} \in \hat{H}^{1}(\mathcal{A} / \mathcal{G})$ does not depend on $\mathfrak{A}$.

Proof. By equation (13) we have $\xi_{c, \underline{A}}([A])=\xi_{c, \underline{A}}(s)+\int_{c \times[A]} T p\left(\underline{\mathfrak{A}}^{\prime}, \underline{\mathfrak{A}}\right)^{2 r-1,0}$. But $T p\left(\underline{\mathfrak{A}}^{\prime}, \underline{\mathfrak{A}}\right)^{2 r-1,0}=0$ because $\underline{\mathfrak{A}}^{\prime}-\underline{\mathfrak{A}} \in \Omega^{0,1}(M \times \mathcal{A} / \mathcal{G}, \operatorname{ad}(P \times \mathcal{A} / \mathcal{G}))$.

As $\xi_{c, \underline{A}} \in \hat{H}^{1}(\mathcal{A} / \mathcal{G})$ does not depend on $\mathfrak{A}$, we denote $\xi_{c, \underline{A}}$ simply by $\xi_{c}$. The first order character $\xi_{c}$ determines a function $\varphi_{c}: \mathcal{A} / \mathcal{G} \rightarrow \mathbb{R} / \mathbb{Z}$ given by $\varphi_{c}([A])=\xi_{c}([A])=\chi_{\mathfrak{A}}(c \times[A])$. The connection $A$ induces a morphism of principal $G$-bundles $g_{A}: P \rightarrow(P \times \mathcal{A}) / \mathcal{G}$, defined by $g_{A}(y)=[(y, A)]$ for $y \in P$. By Proposition 3 we have $g_{A}^{*}(\mathfrak{A})=A$, and hence $\underline{g}_{A}^{*} \chi_{\mathfrak{A}}=\chi_{A}$, where $\underline{g}_{A}: M \rightarrow$ $M \times(\mathcal{A} / \mathcal{G})$ is given by $\underline{g}_{A}(x)=(x,[A])$. We conclude that $\lambda_{c}(A)=\chi_{A}(c)=$ $\underline{g}_{A}^{*} \chi_{\mathfrak{A}}(c)=\chi_{\mathfrak{A}}(c \times[A])=\varphi_{c}([A])$. In particular this proves that $\lambda_{c}$ is $\mathcal{G}$-invariant.

$\bar{W}$ study the restriction to the moduli space of flat connections. Equations (14) and (15) imply that if $A$ is flat then for $r \geq 1$ the Chern-Simons action $\lambda_{c}(A)$ only depends on the homology class of $c$. Also, for $r \geq 2, \lambda_{c}(A)$ only depends on the connected component of $[A]$ on $\mathcal{F} / \mathcal{G}$ (i.e, $\lambda_{c}(A)$ determines a locally constant function on $\mathcal{F} / \mathcal{G}$ ). For example, when $\operatorname{dim} M=3$ and $c=M$ this fact is well known, as the extremals of the Chern-Simons action are the flat connections, and hence the Chern-Simons action is constant on each connected component of $\mathcal{F}$.

\subsection{Differential characters of order 2}

First we recall that if $\mathcal{U} \rightarrow M$ a principal $U(1)$-bundle with connection $\Theta$, and curvature $\omega \in \Omega^{2}(M)$, the log-holonomy of $\Theta \log \operatorname{hol}_{\Theta}: Z_{1}(M) \rightarrow \mathbb{R} / \mathbb{Z}$ is a differential character of order 2 with curvature $\omega$. A classical result in differential cohomology asserts that the converse is true, that is, every second order differential character can be represented as the holonomy of a connection $\Theta$ on a principal $U(1)$ bundle $\mathcal{U} \rightarrow M$ and this bundle and connection are unique modulo isomorphisms. In our case is possible to give a concrete bundle and connection using the following result proved in [19]

Theorem 14 Let $N$ be a connected and simply connected manifold in which $\mathcal{G}$ acts in such a way that $\pi: N \rightarrow N / \mathcal{G}$ is a principal $\mathcal{G}$-bundle. Let $\chi \in \hat{H}^{2}(N / \mathcal{G})$ 
be a second order differential character on $N / \mathcal{G}$ with curvature $\omega$, and assume that there exist $\lambda \in \Omega^{1}(N)$ such that $\pi^{*} \omega=d \lambda$. Then there exists a unique lift of the action of $\mathcal{G}$ to $N \times U(1)$ by $U(1)$-bundle automorphism such that $\Theta=\vartheta-2 \pi i \lambda \in \Omega^{1}(N \times U(1), i \mathbb{R})$ is projectable onto a connection $\underline{\Theta}$ on $\mathcal{U}=$ $(N \times U(1)) / \mathcal{G} \rightarrow N / \mathcal{G}$ and $\chi=\log \operatorname{hol}_{\underline{\Theta}}$ ( $\vartheta$ denotes the Maurer-Cartan form on $U(1))$.

The action of $\phi \in \mathcal{G}$ on $N \times U(1)$ is given by $\Phi_{\phi}: N \times U(1) \rightarrow N \times U(1)$, $\Phi_{\phi}(x, u)=\left(\phi x, \exp \left(2 \pi i \alpha_{\phi}(x)\right) \cdot u\right)$, where $\alpha_{\phi}: N \rightarrow \mathbb{R} / \mathbb{Z}$ is defined by $\alpha_{\phi}(x)=$ $\int_{\gamma} \lambda-\chi(\pi \circ \gamma)$, and $\gamma$ is any curve on $N$ joining $x$ and $\phi x$.

If $c \in Z_{2 r-2}(M)$ we have $\xi_{c, \underline{A}} \in \hat{H}^{2}(\mathcal{A} / \mathcal{G})$. By Section $3 \varpi_{c}=\int_{c} p_{\mathcal{G}, \mathbb{A}} \in$ $\Omega_{\mathcal{G}}^{2}(\mathcal{A} / \mathcal{G})$ can be written $\varpi_{c}=\sigma_{c}+\mu_{c}$, and by the definition of $p_{\mathcal{G}, \mathbb{A}}$ we have $\sigma_{c}=\int_{c} p(\mathbb{F})$ and $\mu_{c}(X)=-r \int_{c} p\left(\operatorname{pr}_{1}^{*} X, \mathbb{F},(\stackrel{r-1}{\cdots}, \mathbb{F})\right.$. If $A_{0}$ is a connection on $P \rightarrow$ $M$, we define $\rho_{c}=\int_{c} T p\left(\mathbb{A}, \overline{\operatorname{pr}}_{1}^{*} A_{0}\right) \in \Omega^{1}(\mathcal{A})$, where $\overline{\mathrm{pr}}_{1}: P \times \mathcal{A} \rightarrow P$ denotes the projection. If we set $\lambda_{c}=\rho_{c}+\mu_{c}(\mathfrak{A})$, we have the following

Proposition 15 We have $\operatorname{curv}\left(\xi_{c, \underline{\mathfrak{A}}}\right)=\int_{c} p(\underline{\mathfrak{F}})$ and $\pi^{*}\left(\int_{c} p(\underline{\mathfrak{F}})\right)=d \lambda_{c}$.

Proof. For any $s \in Z_{1}(\mathcal{A} / \mathcal{G})$ we have $\xi_{c, \underline{\mathfrak{A}}}(\partial s)=\chi_{\underline{\mathfrak{A}}}(c \times \partial s)=\chi_{\underline{\mathfrak{A}}}(\partial(c \times s))=$ $\int_{c \times s} p(\underline{\mathfrak{F}})=\int_{s} \int_{c} p(\underline{\mathfrak{F}})$, and by the definition of the curvature of a differential character we conclude that $\operatorname{curv}\left(\xi_{c, \underline{A}}\right)=\int_{c} p(\underline{\mathfrak{F}})$.

Furthermore, we have $d \rho_{c}=\int_{c} d T p\left(\mathbb{A}, \overline{\operatorname{pr}_{1}^{*}} A_{0}\right)=\int_{c} p(\mathbb{F})-\int_{c} \operatorname{pr}_{1}^{*} p\left(F_{0}\right)=\sigma_{c}$ as $\int_{c} \operatorname{pr}_{1}^{*} p\left(F_{0}\right)=0$ because $\operatorname{pr}_{1}^{*} p\left(F_{0}\right) \in \Omega^{2 r-1,0}$. By equation 6 we have $\int_{c} p(\underline{\mathfrak{F}})=$ $\mathrm{C}_{\mathfrak{A}}\left(\int_{c} p_{\mathcal{G}}\right) \in \Omega_{\mathcal{G}}^{2}(\mathcal{A} / \mathcal{G})$, and the result follows from Lemma 2

By applying Proposition 14 to the bundle $\pi_{\mathcal{A}}: \mathcal{A} \rightarrow \mathcal{A} / \mathcal{G}$ and the character $\xi_{c, \mathfrak{A}}$ we obtain the following

Proposition 16 Let $A_{0}$ be a background connection on $P \rightarrow M$ and $\mathfrak{A}$ be a connection on $\mathcal{A} \rightarrow \mathcal{A} / \mathcal{G}$. Then there exists a unique lift of the action of $\mathcal{G}$ on $\mathcal{A}$ to an action on $\mathcal{A} \times U(1)$ by $U(1)$-bundle automorphisms such that $\Theta_{c}=\vartheta-2 \pi i\left(\rho_{c}+\mu_{c}(\mathfrak{A})\right) \in \Omega^{1}(\mathcal{A} \times U(1), i \mathbb{R})$ is projectable onto a connection $\underline{\Theta}_{c}$ on $\mathcal{U}_{c}=(\mathcal{A} \times U(1)) / \mathcal{G} \rightarrow \mathcal{A} / \mathcal{G}$ and $\xi_{c, \underline{A}}=\log \operatorname{hol}_{\underline{\Theta}_{c}}$.

The action of $\phi \in \mathcal{G}$ on $\mathcal{A} \times U(1)$ is given by $\Phi_{\phi}: \mathcal{A} \times U(1) \rightarrow \mathcal{A} \times U(1)$, $\Phi_{\phi}(A, u)=\left(\phi A, \exp \left(2 \pi i \alpha_{\phi}(A)\right) \cdot u\right)$, where $\alpha_{\phi}: \mathcal{A} \rightarrow \mathbb{R} / \mathbb{Z}$ is defined by $\alpha_{\phi}(A)=$ $\int_{\gamma} \lambda-\chi\left(\pi_{\mathcal{A}} \circ \gamma\right)$, and $\gamma$ is any curve on $\mathcal{A}$ joining $A$ and $\phi A$.

Remark 17 As the characteristic class of the holonomy of a connection coincides with the Chern class of the bundle we also have $c_{1}\left(\mathcal{U}_{c}\right)=\operatorname{char}\left(\xi_{c, \underline{A}}\right)=$ $\Upsilon_{(P \times \mathcal{A}) / \mathcal{G}} / c$ where $\Upsilon_{(P \times \mathcal{A}) / \mathcal{G}} \in H^{2 r}(M \times \mathcal{A} / \mathcal{G}, \mathbb{Z})$ is the characteristic class of $(P \times \mathcal{A}) / \mathcal{G} \rightarrow M \times \mathcal{A} / \mathcal{G}$ associated to $\Upsilon$, and / denotes cap product.

In place of the principal $U(1)$-bundle $\mathcal{U}_{c}$, we can consider the associated Hermitian line bundle $\mathcal{L}_{c}$. The connection $\underline{\Theta}_{c}$ determines a hermitian connection $\nabla \underline{\Theta}_{c}$ on this bundle.

The bundle $\mathcal{U}_{c}$ is defined using a connection $\mathfrak{A}$ in $\mathcal{A} \rightarrow \mathcal{A} / \mathcal{G}$, but we have the following 
Proposition 18 The map $\alpha$ is independent of the connection $\mathfrak{A}$ chosen in $\mathcal{A} \rightarrow$ $\mathcal{A} / \mathcal{G}$.

Proof. If $\mathfrak{A}^{\prime}$ is another connection on $\mathcal{A} \rightarrow \mathcal{A} / \mathcal{G}$ we have $\lambda_{c}^{\prime}=\rho_{c}+\mu_{c}\left(\mathfrak{A}^{\prime}\right)=$ $\lambda_{c}+\mu_{c}\left(\mathfrak{A}^{\prime}-\mathfrak{A}\right)$ and by equation (13) for any curve $\gamma$ on $N$ such that $\pi_{\mathcal{A}}(\gamma(0))=$ $\pi_{\mathcal{A}}(\gamma(1))$ we have $\xi_{c, \underline{\mathfrak{A}}}\left(\pi_{\mathcal{A}} \circ \gamma\right)=\xi_{c, \underline{\mathfrak{A}}}\left(\pi_{\mathcal{A}} \circ \gamma\right)+\int_{c \times \pi_{\mathcal{A}} \circ \gamma} T p\left(\underline{\mathfrak{A}}^{\prime}, \mathfrak{A}^{\prime}\right)=\xi_{c, \mathfrak{A}}(\pi \circ$ $\gamma)+\int_{c \times \gamma} T p\left(\pi_{\mathcal{G}}^{*} \underline{A}^{\prime}, \pi_{\mathcal{G}}^{*} \underline{A}\right)$, where $\pi_{\mathcal{G}}: M \times \mathcal{A} \rightarrow M \times \mathcal{A} / \mathcal{G}$ is the projection. But $T p\left(\pi^{*} \underline{\mathfrak{A}}^{\prime}, \pi^{*} \underline{\mathfrak{A}}\right)=r \int_{0}^{1} p\left(\pi^{*}\left(\underline{\mathfrak{A}}^{\prime}-\underline{\mathfrak{A}}\right), F_{t}, \ldots, F_{t}\right)=r \int_{0}^{1} p\left(\mathbb{A}\left(\mathfrak{A}^{\prime}-\mathfrak{A}\right), F_{t}, \ldots, F_{t}\right)$, where $F_{t}$ is the curvature of $A_{t}=\mathbb{A}-\mathbb{A}\left((1-t) \mathfrak{A}^{\prime}+t \mathfrak{A}\right)$. By Lemma 8 we have $F_{t}^{2,0}=\mathbb{F}^{2,0}$ and hence $\int_{c \times \gamma} T p\left(\pi^{*} \underline{\mathfrak{A}}^{\prime}, \pi^{*} \underline{\mathfrak{A}}\right)=\int_{c \times \gamma} T p\left(\pi^{*} \underline{\mathfrak{A}}^{\prime}, \pi^{*} \underline{\mathfrak{A}}\right)^{1,2 r-2}=$ $\int_{c \times \gamma} r \int_{0}^{1} p\left(\mathbb{A}\left(\mathfrak{A}^{\prime}-\mathfrak{A}\right), \mathbb{F}, \ldots, \mathbb{F}\right)=\int_{\gamma} \mu_{c}\left(\mathfrak{A}^{\prime}-\mathfrak{A}\right)$. The result follows as we have $\alpha_{\phi}^{\prime}(A)=\int_{\gamma} \lambda^{\prime}-\xi_{c, \underline{A}}(\pi \circ \gamma)=\int_{\gamma} \lambda+\int_{\gamma} \mu_{c}\left(\mathfrak{A}^{\prime}-\mathfrak{A}\right)-\xi_{c, \mathfrak{A}}(\pi \circ \gamma)-\int_{\gamma} \mu_{c}\left(\mathfrak{A}^{\prime}-\mathfrak{A}\right)=$ $\alpha_{\phi}(A)$.

These bundles are extended to the case of non-free actions and also to the action of automorphisms in [19]. It is proved that bundles for different $A_{0}$ are canonically isomorphic and that these bundles generalize the Chern Simons lines as if $c=\partial u$, then the Chern-Simons action $S_{u}(A)=\exp \left(-2 \pi i \cdot \int_{u} T p\left(A, A_{0}\right)\right)$ determines a section of $\mathcal{U}_{c}$.

Our interest is in the restriction of $\mathcal{U}_{c}$ to the moduli space of flat connections. For $r \geq 2$ we have $\mathcal{F} \subset \mu_{c}^{-1}(0)$. In particular the restriction $\theta_{c}=\iota_{\mathcal{F}}^{*} \underline{\Theta}_{c}$ to $\mathcal{F} \times U(1)$ of the form $\underline{\Theta}_{c}$ does not depend on $\mathfrak{A}$ as $\mu_{c}(\mathfrak{A})=0$ on $\mathcal{F}$. We conclude that the restriction of the bundle $\mathcal{U}_{c} \rightarrow \mathcal{A} / \mathcal{G}$ and of the connection $\underline{\Theta}_{c}$ do not depend on $\mathfrak{A}$, in accordance with Theorem 9 .

The curvature of $\theta_{c}$ is the pre-symplectic form $\underline{\sigma}_{c}$ on $\mathcal{F} / \mathcal{G}$ obtained by symplectic reduction to $\mathcal{F}$ from $\left(\mathcal{A}, \varpi_{c}\right)$. For example, if $M$ is a closed oriented surface we can take $c=M, G=S U(2), P$ the trivial $S U(2)$-bundle, $p(X)=\frac{1}{8 \pi^{2}} \operatorname{tr}\left(X^{2}\right)$ and $\Upsilon$ the second Chern class then $\sigma_{M}$ and $\mu_{M}$ coincide with the symplectic structure and moment map defined on 2] (e.g. see [16]). Furthermore, it is shown in [19] that the bundle $\mathcal{L}_{M} \rightarrow \mathcal{F} / \mathcal{G}$ is isomorphic to Quillen determinant line bundle. Hence, our character $\xi_{M}$ is the holonomy of a connection $\theta_{M}$ on the determinant line bundle $\operatorname{det} \bar{\partial} \rightarrow \mathcal{F} / \mathcal{G}$.

If $\operatorname{dim} M>2$, for each submanifold $c$ of dimension 2 we have a line bundle $\mathcal{L}_{c} \rightarrow \mathcal{F} / \mathcal{G}$ with connection $\theta_{c}$. A similar family of bundles $\ell_{c} \rightarrow \mathcal{A} / \mathcal{G}$ is constructed for example in [14, Section 5.2.1] associated to surfaces $c$ immersed on a 4-manifold $M$. These bundles are defined as determinant line bundles of Dirac operators, and are isomorphic to our bundles as they have the same integer Chern class $c_{1}\left(\ell_{c}\right)=\Upsilon_{(P \times \mathcal{A}) / \mathcal{G}} / c=c_{1}\left(\mathcal{L}_{c}\right) \in H^{2}(\mathcal{A} / \mathcal{G}, \mathbb{Z})$.

From equation (15) we conclude that the holonomy of the connection $\theta_{c}$ only depends on the homology class of $c$.

If $r \geq 3$ we have $\left.\sigma_{c}\right|_{\mathcal{F}}=0$, and in this case $\theta_{c}$ is a flat connection on $\mathcal{L}_{c} \rightarrow$ $\mathcal{F} / \mathcal{G}$, and hence its holonomy defines a cohomology class in $H^{1}(\mathcal{F} / \mathcal{G}, \mathbb{R} / \mathbb{Z})$, in accordance with our result of equation (14). Furthermore this equation implies that the cohomology class only depends on the homology class of $c$ on $M$. 


\section{Particular cases}

The main objects $\chi^{k}$ of this work are close to some constructions found in the literature:

The case $k=\operatorname{dim} M-2 r+1$. If we choose a cohomology class $c \in H_{2 r-k-1}(M)$, for $k \leq r-1$ we obtain cohomology classes $\xi_{c} \in H^{k}(\mathcal{F} / \mathcal{G}, \mathbb{R} / \mathbb{Z}), \xi_{c}(s)=\chi^{k}(c, s)$. When $M$ is a compact manifold without boundary of dimension $\operatorname{dim} M=$ $2 r-k-1$, for $k \leq r-1$, the cohomology classes $\xi_{M}$ coincide with some classes defined on [15. In that article, the authors work with families of connections instead of $\mathcal{A} / \mathcal{G}$, and Deligne cohomology instead of differential characters. Note

that our result is more general in the sense that we obtain cohomology classes also for submanifolds $c \subset M$.

The unitary group. For $G=U(n)$, we consider the map (14) for the choice of $p_{r}$ and $\mu_{r}$ as $r$-th Chern polynomial and class, $k \leq r-2$, respectively. Furthermore, we regard that map in the cohomology level as

$$
\left.\chi_{p_{r}}^{k}\right|_{\mathcal{F} / \mathcal{G}}: H_{k}(\mathcal{F} / \mathcal{G}) \rightarrow H^{2 r-k-1}(M, \mathbb{R} / \mathbb{Z})
$$

By adding the maps for different $r$, we obtain the map $\tilde{\chi}^{k}=\left.\bigoplus_{r=k+2}^{n} \chi_{p_{r}}^{k}\right|_{\mathcal{F} / \mathcal{G}}$,

$$
\tilde{\chi}^{k}: H_{k}(\mathcal{F} / \mathcal{G}) \rightarrow \bigoplus_{r=k+2}^{n} H^{2 r-k-1}(M, \mathbb{R} / \mathbb{Z})
$$

which is a similar to the construction in 22, by a completely different method, for the study of cohomological invariants of variations of flat connections.

\section{References}

[1] B. Antieau and B. Williams, The Topological Period-Index Problem over 6-Complexes, J. Top. 7 (2014), 617-640.

[2] M. F. Atiyah, R. Bott, The Yang-Mills equations over Riemann surfaces, Phil. Trans. R. Soc. Lond. A 308 (1982) 523-615.

[3] M.F. Atiyah, I. Singer, Dirac operators coupled to vector potentials, Proc. Natl. Acad. Sci. USA 81 (1984), 2597-2600.

[4] C. Bär, C. Becker Differential Characters. Lecture Notes in Math. 2112, Springer, Berlin 2014.

[5] N. Berline, M. Vergne, Classes caractéristiques équivariantes. Formules de localisation en cohomologie équivariante, C. R. Acad. Sci. Paris 295 (1982) $539-541$. 
[6] —, Zéros d'un champ de vecteurs et classes charactéristiques équivariantes, Duke Math. J. 50 (1983) 539-549.

[7] N. Berline, E. Getzler, M. Vergne, Heat Kernels and Dirac Operators, Springer Verlag Berlin Heidelberg 1992.

[8] I. Biswas, M. Castrillón López, Flat connections and cohomology invariants, to appear in Math. Nach.

[9] R. Bott, L. Tu, Equivariant characteristic classes in the Cartan model, Geometry, analysis and applications (Varanasi, 2000), 3-20, World Sci. Publishing, River Edge, NJ, 2001.

[10] M. Castrillón López, J. Muñoz Masqué, The geometry of the bundle of connections, Math. Z. 236 (2001), 797-811.

[11] S-S. Chern, J. Simons, Characteristic forms and geometric invariants, Ann. Math. 99 (1974), 48-69.

[12] J. Cheeger and J. Simons, Differential characters and geometric invariants, in Geometry and Topology, Proc. Spec. Year, College Park/Md. 1983/84, Lecture Notes in Math. 1167, Springer-Verlag, Berlin-Heidelberg-New York, 1985.

[13] R. Dijkgraaf and E. Witten. Topological Gauge Theories and Group Cohomology, Commun. Math. Phys., 129 (1990) 393-429.

[14] S.K. Donaldson, P.B. Kronheimer, The geometry of four-manifolds. Oxford University Press, 1990.

[15] J.L. Dupont and F. Kamber, Gerbes, simplicial forms and invariants for families of foliated bundles, Comm. Math. Phys. 253 (2005) 253-282.

[16] R. Ferreiro Pérez, Equivariant characteristic forms in the bundle of connections, J. Geom. Phys. 54 (2005), 197-212

[17] —, Local cohomology and the variational bicomplex, Int. J. Geom. Methods Mod. Phys., 05 (2008) 587-604.

[18] —, Local anomalies and local equivariant cohomology, Comm. Math. Phys. 286 (2009) 445-458.

[19] -, Equivariant prequantization bundles on the space of connections and characteristic classes, preprint arXiv:1703.05832.

[20] D. S. Freed, Classical Chern-Simons theory, Part 2, Houston J. Math. 28 (2002) 293-310.

[21] V. Guillemin, S. Sternberg, Supersymmetry and Equivariant de Rham Theory, Springer 1999. 
[22] Iyer, J.N.N. Cohomological Invariants of a Variation of Flat Connections, Lett Math Phys 106 (2016) : 131-146.

[23] D. Krupka, The contact ideal, Differential Geom. Appl. 5 (1995), 257-276.

\section{(M.C.L) ICMAT (CSIC-UAM-UC3M-UCM)}

Departamento de Álgebra, Geometría y Topología, Facultad de Matemáticas, Universidad Complutense de Madrid, 28040-Madrid, SPAIN

E-mail: mcastri@mat.ucm.es

(R.F.P) Departamento de Economía Financiera y Contabilidad I. FaCultad de Ciencias Económicas y Empresariales, Universidad ComPLUTENSE DE MADRID, 28223-MADRID, SPAIN

E-mail: roferreiro@ccee.ucm.es 